\title{
Dynamic Reorganization of Digit Representations in Somatosensory Cortex of Nonhuman Primates after Spinal Cord Injury
}

\author{
Li Min Chen, ${ }^{1,2}$ Hui-Xin Qi, ${ }^{3}$ and Jon H. Kaas ${ }^{1,3}$ \\ ${ }^{1}$ Department of Radiology and Radiological Sciences, ${ }^{2}$ Institute of Imaging Science, and ${ }^{3}$ Department of Psychology, Vanderbilt University, Nashville, \\ Tennessee 37240
}

\begin{abstract}
Somatosensory cortices of adult primates reactivate over time after sensory loss. The time course and the neural mechanisms underlying the cortical reactivation are not well understood. Here we report that longitudinal high-resolution functional magnetic resonance imaging (fMRI) studies on anesthetized squirrel monkeys revealed dynamic reorganizations of digit activations in area $3 \mathrm{~b}$, within 2 months after severely disrupting afferent inputs by dorsal column section. We found that digit regions in which inputs were severely disrupted exhibited fMRI tactile responses. Reorganization was characterized by an early moving away phase and a late returning phase, as indicated by spatial shifts of individual digit activation centers in relation to the pre-lesion activation sites. Subsequent optical imaging studies confirmed fMRI activations, and dense microelectrode penetrations identified weak neuronal activity at the reactivated sites. Activation zones detected by fMRI and optical imaging were significantly larger in input-deprived than normal input single-digit regions and were larger than regions defined by neuronal spiking activity. This study captures the dynamic reorganization of digit representations after dorsal column lesions and reveals differences between functional imaging and microelectrode recording maps. Our observations suggest that subthreshold activity plays an important role in the reactivation of deafferented cortex and could promote behavioral recovery.
\end{abstract}

\section{Introduction}

The ability to monitor longitudinally plastic changes in the nervous system after damage (such as after spinal cord injury) is essential for understanding the neuronal mechanisms that mediate neural and behavioral recovery. Plastic changes after spinal cord injuries exist at multiple relay stations along the ascending pathway (for review, see Buonomano and Merzenich, 1998; Jones, 2000; Kaas et al., 2008). These plastic changes correlate with behavioral outcomes (Almli and Finger, 1988; DarianSmith and Ciferri, 2005; Padberg et al., 2010; Qi et al., 2011). Currently, our ability to gain a detailed understanding of the functional role of plastic remodeling in the nervous system is limited by the lack of effective tools for monitoring these changes

Received April 16, 2012; revised July 27, 2012; accepted Aug. 17, 2012.

Author contributions: L.M.C., H.-X.Q., and J.H.K. designed research; L.M.C. and H.-X.Q. performed research; L.M.C. and H.-X.Q. contributed unpublished reagents/analytic tools; L.M.C. and H.-X.Q. analyzed data; L.M.C., H.-X.Q., and J.H.K. wrote the paper.

This work is supported by a Dana Foundation Grant (L.M.C.), National Institutes of Health Grants NS057399 and NS067017 (H.-X.Q.) and NS16446 (J.H.K.), and an award from the Dana and Christopher Reeve Foundation (J.H.K.). We thank Drs. Barbara C. Dillenburger, Feng Wang, and Omar A. Gharbawie for their assistance in data collection and Chaohui Tang for her excellent technical support on animal preparation. We also thank Dr. Anna Roe for allowing us to use her electrophysiology and optical imaging equipment. We declare that our research was conducted in accordance with the Statement of Ethical Standards. Vanderbilt University Institutional Animal Care and Use Committee approved all the procedures described in this manuscript.

The authors declare no competing financial interests.

Correspondence should be addressed to Dr. Li Min Chen, Institute of Imaging Science, Department of Radiology and Radiological Sciences, Vanderbilt University Medical Center, 1161 21st Avenue South, Nashville, TN 37027. E-mail: limin.chen@vanderbilt.edu.

DOI:10.1523/JNEUROSCI.1841-12.2012

Copyright $\odot 2012$ the authors $\quad 0270-6474 / 12 / 3214649-15 \$ 15.00 / 0$ noninvasively. Because of its capability of measuring functional organization in the brain non-invasively and longitudinally, functional magnetic resonance imaging (fMRI) has become one of the most commonly used tools for clinical and basic neuroscience research (Duong et al., 2001; Harel et al., 2006, 2012; Matthews et al., 2006). Submillimeter fMRI is now routinely achievable in nonhuman primates (Logothetis et al., 1999; Fukuda et al., 2005, 2006; Chen et al., 2007).

There is compelling evidence that, after partial or nearly complete damage to the nervous system (Gilman and Denny-Brown, 1966; Leonard et al., 1992; Kaas, 2000), mature humans and other mammals typically demonstrate various degrees of behavioral recovery over a period of days to months (Finger and Almli, 1985; Jain et al., 1997). These functional and behavioral recoveries are correlated with functional reorganization of the nervous system in various clinical diseases and experimental conditions (Flor et al., 1995, 1997; Mühlnickel et al., 1998; Karl et al., 2001; Muellbacher and Hallett, 2006; Fox 2009), although the neuronal mechanisms underlying plastic changes in the brain and behavioral recoveries remain unclear (for discussion, see Jain et al., 2008; Graziano and Jones, 2009). A partial unilateral dorsal column lesion has proven to be an excellent model system for the study of brain reorganization and functional recovery (Cooper et al., 1993; Jain et al., 1997; Darian-Smith and Ciferri, 2006; Kaas et al., 2008; Qi et al., 2011) and has shown that the reactivation of area $3 \mathrm{~b}$, along with other cortical and subcortical regions (Jain et al., 1997, 2008), contribute significantly to the functional and behavioral recovery of the damaged sensorimotor functions. Thus far, however, no studies have directly examined the tempo- 
ral relationship between cortical reorganization and functional and behavioral recovery. To this end, we longitudinally evaluated the dynamic changes in responsiveness of somatosensory cortex to tactile stimuli before and after dorsal column lesion with repeated submillimeter resolution fMRI. Our first report from this series of studies focused on the relationship between the responsiveness of areas $3 \mathrm{~b}$ and 1 and the remaining afferent fibers after dorsal column section (Qi et al., 2011). Here we report the longitudinal fMRI findings as well as the validation with optical imaging $(\mathrm{OI})$ and dense microelectrode mapping for the same group of monkeys.

\section{Materials and Methods}

Eight male squirrel monkeys (four spinal-cord-lesioned and four normal controls for a total of 11 hemispheres) were included in this study. This report focuses on the longitudinal changes in the fMRI and OI maps for individual digit activations after dorsal column lesion. The corresponding histological evaluations of the levels and spatial extents of the spinal cord lesions in these animals have been reported in detail in our previous study (Qi et al., 2011).

Animal preparation. Of the four spinal-cord-lesioned monkeys, a total of five pre-lesion and 11 post-lesion scans were obtained from the lesioned animals. For the fMRI experiments, each monkey was initially sedated with ketamine hydrochloride $(10 \mathrm{mg} / \mathrm{kg}) /$ atropine $(0.05 \mathrm{mg} / \mathrm{kg})$ and then maintained on mechanical ventilation with isoflurane anesthesia $(0.5-1.1 \%)$ delivered in a $70: 30 \mathrm{O}_{2} / \mathrm{N}_{2} \mathrm{O}$ mixture. After intubation, the animal was placed in a custom-designed MR cradle, and the head was secured with ear and head bars. Dextrose at $2.5 \%$ in saline was infused intravenously $\left(3 \mathrm{ml} \cdot \mathrm{kg}^{-1} \cdot \mathrm{h}^{-1}\right)$ throughout the imaging session to prevent dehydration and provide caloric energy. Oxygen saturation rate and heart rate (Nonin), ECG, end-tidal $\mathrm{CO}_{2}$ (22-26 mmHg; Surgivet), and respiratory patterns (SA Instruments) were externally monitored. Rectal temperature was monitored (SA Instruments) and maintained at 37.5$38.5^{\circ} \mathrm{C}$ by means of a circulating water blanket (Gaymar Industries). Vital signs were monitored throughout the procedure, from induction of anesthesia until full recovery. Standard procedures were used in the fMRI and electrophysiological studies, as published previously (Chen et al., $2007,2009)$. All procedures were in compliance with and approved by the Institutional Animal Care and Use Committee of Vanderbilt University.

Spinal cord section. Dorsal column section of the spinal cord was performed under general anesthesia. Each monkey was initially sedated with ketamine hydrochloride ( $15 \mathrm{mg} / \mathrm{kg}$, i.m.) and then maintained at a surgical level of anesthesia with 1-3\% isoflurane. The depth of anesthesia was evaluated by the heart and respiration rates and testing for withdraw reflexes. Rectal body temperature was maintained at $37-38.5^{\circ} \mathrm{C}$. Under aseptic conditions, a portion of the cervical spinal cord was exposed, and the dorsal columns were sectioned on one side with a fine pair of surgical scissors at cervical level C4-C6. The dura was replaced with Gelfilm and covered with Gelfoam. The opening was closed, and the skin was sutured. Monkeys were carefully monitored until they were fully recovered from anesthesia and then returned to their home cage. The monkeys also received antibiotics and analgesics for $3 \mathrm{~d}$ after surgery. Animals' cage behavior and food intake typically returned to normal shortly after surgery. Additional details about surgical procedures can be found in previous publications from the laboratory (Jain et al., 1997, 2008; Qi et al., 2011).

Stimulus protocol. To ensure a reproducible delivery of stimuli, fingers were secured by gluing small pegs to the fingernails and fixing these pegs firmly in Plasticine, leaving the glabrous surfaces available for vibrotactile stimulation. An innocuous $8 \mathrm{~Hz}$ vibrotactile stimulus was delivered on individual distal finger tips separately, by vertical indentation of the skin (0.48 $\mathrm{mm}$ displacement) with a rounded plastic probe ( $2 \mathrm{~mm}$ in diameter) mounted on a piezoceramic actuator (Noliac) driven by a Grass Instruments stimulator. Vibrotactile stimuli were presented for $30 \mathrm{~s}$ (for fMRI experiments) or $5 \mathrm{~s}$ [for optical imaging (OI) experiments] duration blocks. During off blocks ( $30 \mathrm{~s}$ for fMRI and $7 \mathrm{~s}$ for OI), the probe was lightly touching the skin. Typically, each stimulus condition (e.g., D1) was repeated 14-21 times within a single fMRI imaging session. Because of the goal of mapping all five digits and the narrow space be- tween digit tips, only three digits were stimulated alternately at each time. However, maps of all five digits were acquired in a single imaging session from three lesioned animals (SM-D, SM-C, SM-O) and in two imaging sessions (on two separate days) from the fourth lesioned animal (SM-R). Digit maps in normal control animals (SM-M and SM-F) were all collected in a single imaging session. The MR scanner controlled the stimulus timing by sending trigger pulses to the stimulator to start each stimulus epoch within one run. For the OI experiments, each $5 \mathrm{~s}$ tactile stimulation run was followed by an $8 \mathrm{~s}$ resting period. Fifty trials were typically collected for each stimulus condition (e.g., D1).

MRI methods. All MRI scans were performed on a $9.4 \mathrm{~T} 21-\mathrm{cm}$ narrowbore Varian Inova magnet (Varian Medical Systems) using a $3 \mathrm{~cm}$ surface transmit-receive coil centered over the primary somatosensory cortex (SI) contralateral to the stimulated hand. Scout coronal images ( 15 slices) using a gradient echo sequence were used to plan oblique structural and functional slices. Because the goal of this study was to map tactile responses in SI, four 2 -mm-thick oblique image slices with an in-plane resolution of $0.55 \times 0.55$ $\mathrm{mm}^{2}$ were centered over the central sulcus to maximize the mapping power. Although the cortical areas along the lateral sulcus were also covered by this imaging volume, we only described the findings from SI cortex (the top slice), because the focus of this report is on areas $3 \mathrm{~b}$ and 1 . On the top oblique slice, large- and medium-sized surface veins (Fig. 1, black lines) as well as the penetrating transcortical veins (Fig. 1, black dots) were clearly visible on the $\mathrm{T} 2{ }^{*}$-weighted gradient echo structural image [repetition time (TR), $200 \mathrm{~ms}$; echo time (TE), $14 \mathrm{~ms} ; 512 \times 512$ matrix; $78 \times 78 \times 2000 \mu \mathrm{m}^{3}$ resolution]. Both the surface and transcortical blood vessel features were used for coregistration of MRI maps with surface blood vessel maps obtained later during OI and microelectrode mapping/recording sessions, as well as for MRI maps obtained across imaging sessions conducted on different days. fMRI data were acquired from the same slices using a gradient echo planar imaging sequence (TE, $16 \mathrm{~ms} ; 64 \times 64$ matrix; $0.55 \times 0.55 \times 2 \mathrm{~mm}^{3}$ resolution). A TR of $1.5 \mathrm{~s}$ for individual scans was set to match the ventilation rate to minimize respiration-induced signal variations in BOLD signal time courses.

$f M R I$ data analyses. Reconstructed images were imported into MATLAB (MathWorks) for analysis. The time courses of the BOLD (blood oxygenation level dependent) fMRI data were drift-corrected using a linear model fitted to each time course and temporally smoothed with a low-pass filter whose cutoff frequency was set at $0.25 \mathrm{~Hz}$. No spatial smoothing was applied. The voxelwise correlation of the functional time courses to a boxcar function was calculated. The boxcar predictor was temporally shifted (two image frames) to compensate for the hemodynamic response delay. To increase the signal-to-noise ratio, multiple runs of the same stimulus condition (e.g., D1 activation) were averaged to generate one activation map for each digit. Only runs with significances of correlation coefficiency of $p \leq 10^{-4}$ (converted from $t$ values, uncorrected for multiple comparison; or $p \leq 0.001$ with Bonferroni's correction) were included in averaging. To define a unit of activation, an adaptive threshold was applied to each average map (details are described by Zhang et al., 2010). The cluster threshold was set as two voxels. Specifically, the averaged activation map for each digit was thresholded at a $1 e^{-2}$ lower $p$ value (e.g., $10^{-4}$ ) than its peak $p$ value (e.g., from $10^{-6}$ ). Thresholded single-digit activation maps were interpolated to the native structural resolution $\left(78 \times 78 \mu \mathrm{m}^{2}\right.$ in $512 \times 512$ matrix $)$ and overlaid on high-resolution structural images for display. Based on the thresholded average activation maps, we localized activation centers based on the highest $p$ values (most significant) among the activated voxels and calculated the sizes of activations.

OI of intrinsic signals. OI methods have been described in detail previously (Chen et al., 2001, 2003). In brief, images were collected using the Imager 3001 system (Optical Imaging) and $630 \mathrm{~nm}$ illumination. A blood vessel map, used for landmark purposes, was collected with $570 \mathrm{~nm}$ illumination. In blocks of trials, stimuli were presented in a randomly interleaved manner. A total of 50 trials were collected per stimulus condition. Only single-condition maps, which indicate the response magnitude at each location in the image for a particular stimulus condition, were presented here. The functional maps presented and quantified here are single-condition maps obtained by subtracting the first two prestimulus frames from each subsequent frame of the stimulus condition 


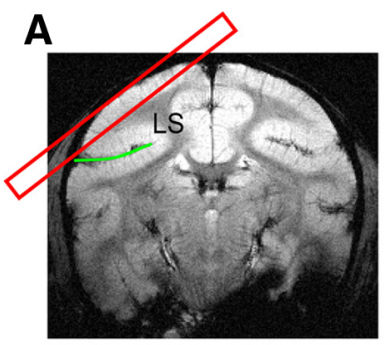

B
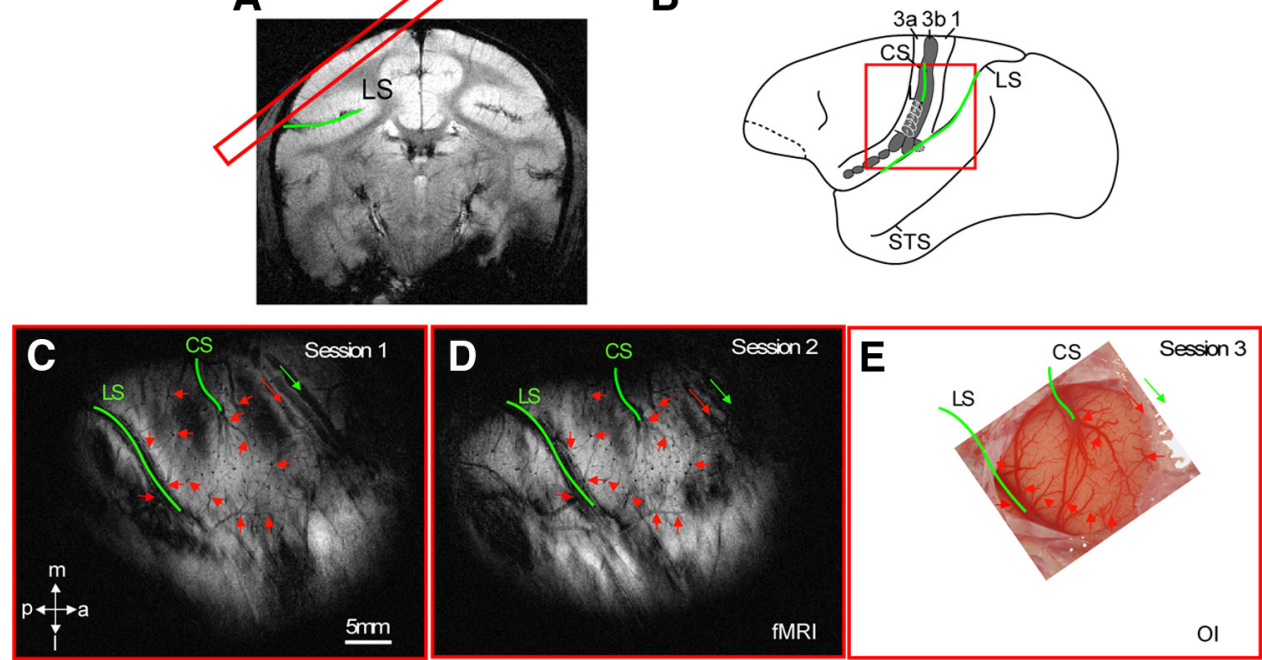

Figure 1. Methods for longitudinal fMRI and cross-model imaging alignment. $A$, The placement of the topmost (red box) of the four oblique image slices, which covers the cortical region around the central sulcus (CS), is shown on coronal image. $\boldsymbol{B}$, Field of view of the top oblique image slice is indicated by the red box placed on top of the schematic illustration of the functional organization of somatosensory areas (areas 3a, 3b, and 1) around the CS. LS, Lateral sulcus; STS, superior temporal sulcus. C, D, T2-weighted structural MRl images obtained from two separate imaging sessions. (S and LS (green lines), surface and trans-cortical veins (black lines and dots) were used as landmarks (red and green arrows) for image alignment. $E$, Corresponding blood vessel map of the image field of view obtained from the same animal. Corresponding alignment landmarks are also shown. This blood vessel map was used for both optical imaging (OIS) and electrophysiology experiments. a, Anterior; l, lateral; m, medial; p, posterior.

(first-frame subtracted). The blank condition is a $5 \mathrm{~s}$ series of images acquired during the no-stimulus condition. Such first-frame subtractions not only measure changes from baseline but also reduce blood vessel artifacts and minimize effects of uneven illumination. In singlecondition maps, dark pixels indicate a response greater than that of the prestimulus image, and gray pixels indicate a response no different from the prestimulus image. For each stimulus condition, image frames were summed to maximize the signal-to-noise ratio. A thresholding procedure was used to delineate the regions of strongest activation. To identify regions of strongest activation, single-condition maps were thresholded at the top $15 \%$ of the gray pixel values distribution. Once the hot spots for activations were outlined using the thresholding method as described above, the total number of pixels outlined was calculated with the freeaccess image-based quantification NIH Image J program (http://rsbweb. nih.gov/ij/). Finally, the total pixel number was converted into square millimeters based on the image field of view.

Quantification of activation shift and size of $\mathrm{fMRI}$ activation. For $\mathrm{AMRI}$ data, after 2D alignment of MRI images obtained from the same cortex on different days, we quantified the distance (in millimeters) between the centers of the same digit activations obtained before spinal cord lesion, and at different recovery points after the lesion, to quantify the spatial shift of single-digit activations after dorsal column section. If there were multiple activation foci within area $3 \mathrm{~b}$ in response to single-digit stimulation, only one measure was taken from the closest activation foci to the pre-lesion activation within area $3 \mathrm{~b}$. In cases in which both area $3 \mathrm{a}$ and area 1 (as well as other cortical area) activations were present, the inter-center distance measure was only taken in area $3 \mathrm{~b}$. Area $3 \mathrm{~b}$ and inter-area borders were determined by the BOLD somatotopic organization of single-digit activations and subsequent dense microelectrode electrophysiological mapping. To maintain the highest spatial resolution possible and retain the unique activation pattern in each animal, we did not coregister activations across animals. The NIH Image J program was used to measure the sizes of each single-digit activation detected in square millimeters.

For OI data, a similar normalized thresholding procedure was used to quantify activation center shifts of individual digits and their sizes. Single-condition first-frame subtracted activation maps were thresholded at the top $15 \%$ of the grayscale distribution of the image pixels for display and quantification. The center of the mass defines the center of single-digit activation. The size of each activation was measured with the NIH Image J program.

Quantification of activation overlap. To quantify the spatial correspondence of single-digit activations defined by different mapping methods of fMRI, OI, and microelectrode mapping, we first calculated the number of pixels within each identified activation focus with each method in the NIH Image J program. We then calculated the percentage overlap between the functional imaging maps (fMRI and OI) and the electrophysiologically defined activation maps of the same digit, by dividing the number of overlapping pixels by the number of electrophysiologically defined activated pixels and then multiplying times $100 \%$. The higher the percentage of the overlap, the closer the spatial correspondence between the functional imaging map and the electrophysiology map.

Alignment of cross-session BOLD fMRI and blood vessel maps. This multi-model and longitudinal study required image registration among three types of images: structural MRI images across imaging sessions (MRI-MRI), digital blood vessel images to structural MRI image alignments, and histology slices to blood vessel images. For the first two types of registration, we identified corresponding anatomical and blood vessel landmarks in each structural image, such as the visible surface vessels and trans-cortical veins; these coordinates were then put into a point-based registration algorithm (implemented in MATLAB; for details, see Chen et al., 2007). The registration transformation between these two sets of coordinates was then applied to one activation image, thereby coregistering the fMRI activation map to the structural MRI image (Hill et al., 1991; Chen et al., 2002). The same principles were applied to MRI-blood vessel coregistration. These landmarks in both structural MRI and blood vessel images were selected without a priori knowledge of the locations of functional activations.

Identification of cortical areas: intracortical microelectrode mapping and recording. The microelectrode mapping results were related to areas $3 \mathrm{a}$, $3 \mathrm{~b}$, and 1 as defined architectonically in brain sections stained for myelin (Gallyas, 1979), as explained in our previous study (Qi et al., 2011). Area $3 \mathrm{~b}$ was recognized by its dense myelination, and septa within its myelin pattern marked the border of the hand with the face representation (Jain et al., 2001). The microelectrode mapping results were consistent with this definition of cortical areas. Areas 3 a and 1 are defined as strips of less myelinated cortex immediately rostral (3a) or caudal (1) to area $3 b$.

\section{Results}

Stable digit fMRI activation maps in normal control animals We longitudinally mapped fMRI digit activations to vibrotactile stimulation of distal finger tips in area $3 \mathrm{~b}$, in both normal and spinal-cord-lesioned animals, by using our recently developed submillimeter resolution fMRI mapping method (Chen et al., 


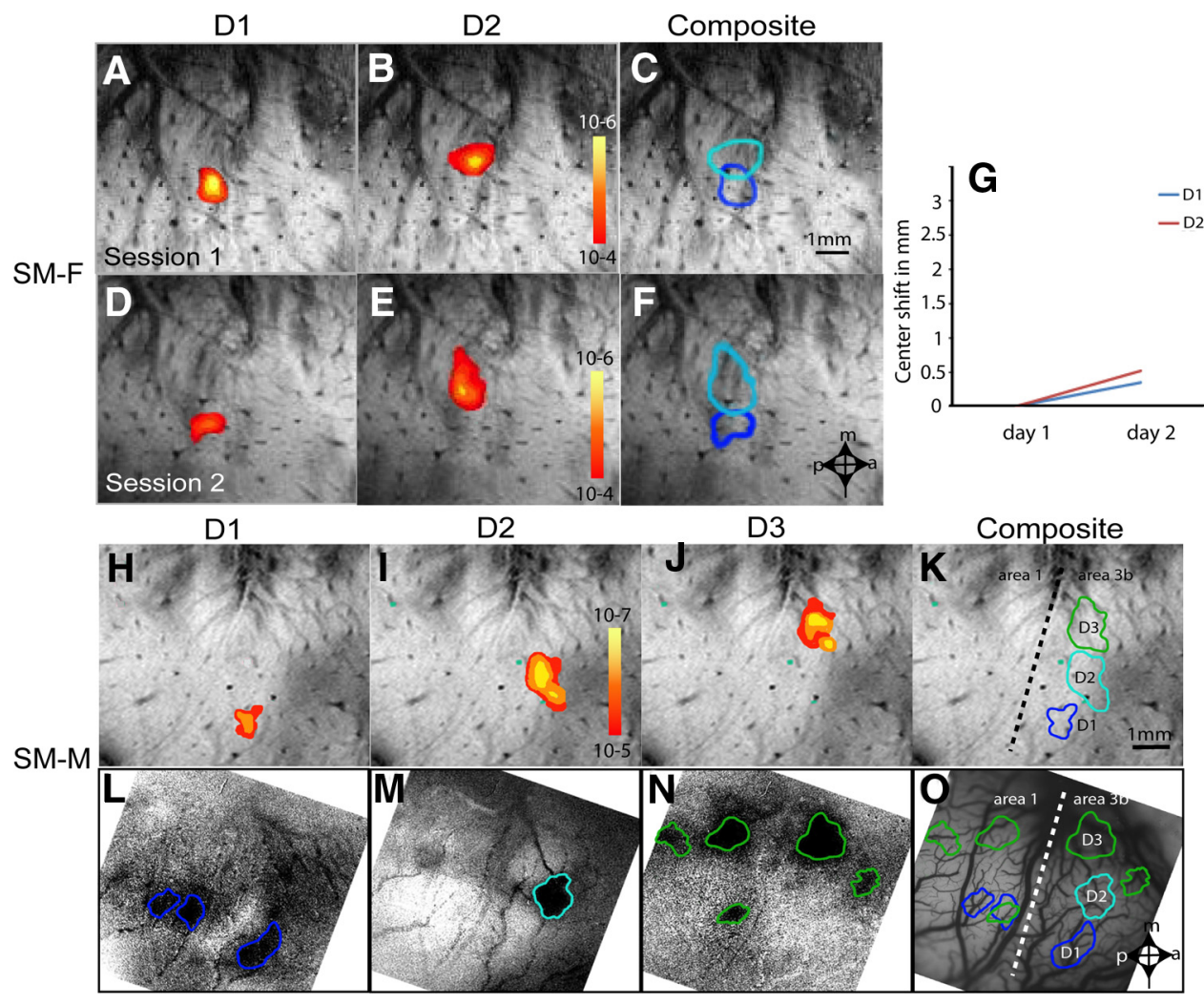

Figure 2. Stable fMRI activation maps of digits and spatial correspondence between $\mathrm{fMRI}$ and 01 maps in normal animals. $A, B$, fMRI activations of D1 and D2 in area $3 \mathrm{~b}$ in response to tactile stimulation on day 1 in monkey SM-F. Color scales indicate the correlation $p$ values of fMRI signal change, thresholded at $p \leq 10^{-4}$. C, Color-coded composite fMRI activation maps of D1 (dark blue outline) and D2 (light blue outline) on day 1.D, E, fMRl activations of the same D1 and D2 to tactile stimulation in area 3b on day 2, 2 months apart from day 1. $\boldsymbol{F}$, Color-coded composite fMRI activation maps of D1 (dark blue outline) and D2 (light blue outline) on day 2. G, Plot of activation center shifts (in millimeters) of each digit as a function of time after lesion. $\boldsymbol{H}-\boldsymbol{J}$, fMRI activations of D1-D3 in the second normal monkey SM-M. Color scale indicates the correlation $p$ values of fMRI signal change. $\boldsymbol{K}$, Composite maps of three digit activations showing orderly somatotopic organization in area $3 \mathrm{~b}$. $\mathbf{L}-\mathbf{N}$, Corresponding 0 activations of D1-D3 in response to identical tactile stimulation. $\mathbf{0}$, Composite map showing the functional organization of the digits that are in close agreement with the fMRl activation map (K). Scale bar, $1 \mathrm{~mm}$. a, Anterior; I, lateral; $\mathrm{m}$, medial; p, posterior.

2007, 2011; Zhang et al., 2010; Lecoeur et al., 2011). Figure 1 summarizes the main technical aspects of the high-resolution fMRI imaging acquisition (Fig. $1 A$ ) and cross-session alignment method (Fig. $1 C, D$ ), which is based on surface and transcortical blood vessel landmarks (green lines and red arrows). fMRI data were later validated by even higher-resolution OI; a photograph of the cortical surface blood vessel pattern that was used for constructing a dense microelectrode electrophysiology map is shown in Figure $1 E$ (electrode penetration sites are shown for this and other cases by Qi et al., 2011). Two scans that were conducted 2 months apart in one representative animal revealed robust and stable single-digit activations to natural tactile stimulation (Fig. $2 A, B, D, E$ ). Digit somatotopy (shown in composite maps in Fig. $1 C, F)$ remained stable over a period of 2 months. Consistent with our previous report (Zhang et al., 2010), the cross-session spatial shifts of digit activation centers were 0.35 and $0.52 \mathrm{~mm}$ for D1 and D2, respectively (Fig. 2G). When we took into account measures from all input-intact digits $(n=12$, including digits from both normal animals and the ipsilateral hemispheres of lesioned animals), the average \pm SD activation center shift was $0.46 \pm 0.05$ $\mathrm{mm}$ (Fig. 12E, left group of columns). Additionally, OI activation (dark patches) maps of D1-D3 in area $3 \mathrm{~b}$ (Fig. $2 L-O$, right) corresponded spatially very well with the fMRI activation maps (Fig. $2 \mathrm{H}-\mathrm{K}$ ). Together, these data indicate that the submillimeter resolution of fMRI at 9.4 T is capable of monitoring quantitative changes in the functional representations of digits in somatosensory area $3 \mathrm{~b}$.

\section{Massive reorganization of digit representations after nearly complete dorsal column lesion}

To evaluate the effects of dorsal column lesion on the functional organization of digits, we mapped longitudinal fMRI responses of all five digits to tactile stimuli in somatosensory area $3 \mathrm{~b}$, before and after lesion at a high cervical level. The extent of somatotopic reorganization of digit representation varied slightly from animal to animal, depending on the level and extent of the dorsal column lesion. Two representative datasets from one severely injured (99\% input deprivation, SM-O) and one less severely injured (76\% input deprivation, SM-D) animal are presented in detail in Figures 3 and 4. For monkey SM-O, before dorsal column lesion, activations of D1-D5 (Fig. 3: D1, blue patch; D2, light blue patch; D3, violet patch; D4, yellow patch; D5, green patch) had an orderly organization in both area $3 \mathrm{~b}$ (left half field of view) and area 1 (right half field of view) in SM-O (Fig. $3 A-F$ ). Four weeks after a high-level (C4) lesion that eliminated $99 \%$ of the entire digit inputs (Fig. 3T; for details, see Qi et al., 2011), focal activations to the $8 \mathrm{~Hz}$ vibrotactile stimulation of the glabrous D1-D5 distal tips were detected (Fig. $3 G-K$ ). In contrast to the orderly D1-D5 activations before the lesion, locations of all digit activations shifted significantly toward the palm regions between area $3 \mathrm{~b}$ and area 1, without a clear somatotopic order (Fig. $3 L$ ). For instance, compared with their pre-lesion activation locations in area $3 \mathrm{~b}$ (Fig. 3F), D1 activation (Fig. 3L, blue patch) shifted anteriorly, whereas D2 (Fig. $3 L$, light blue patch) and D3 (Fig. $3 L$, violet patch) activations shifted laterally in area 3b. D4 activation (Fig. 

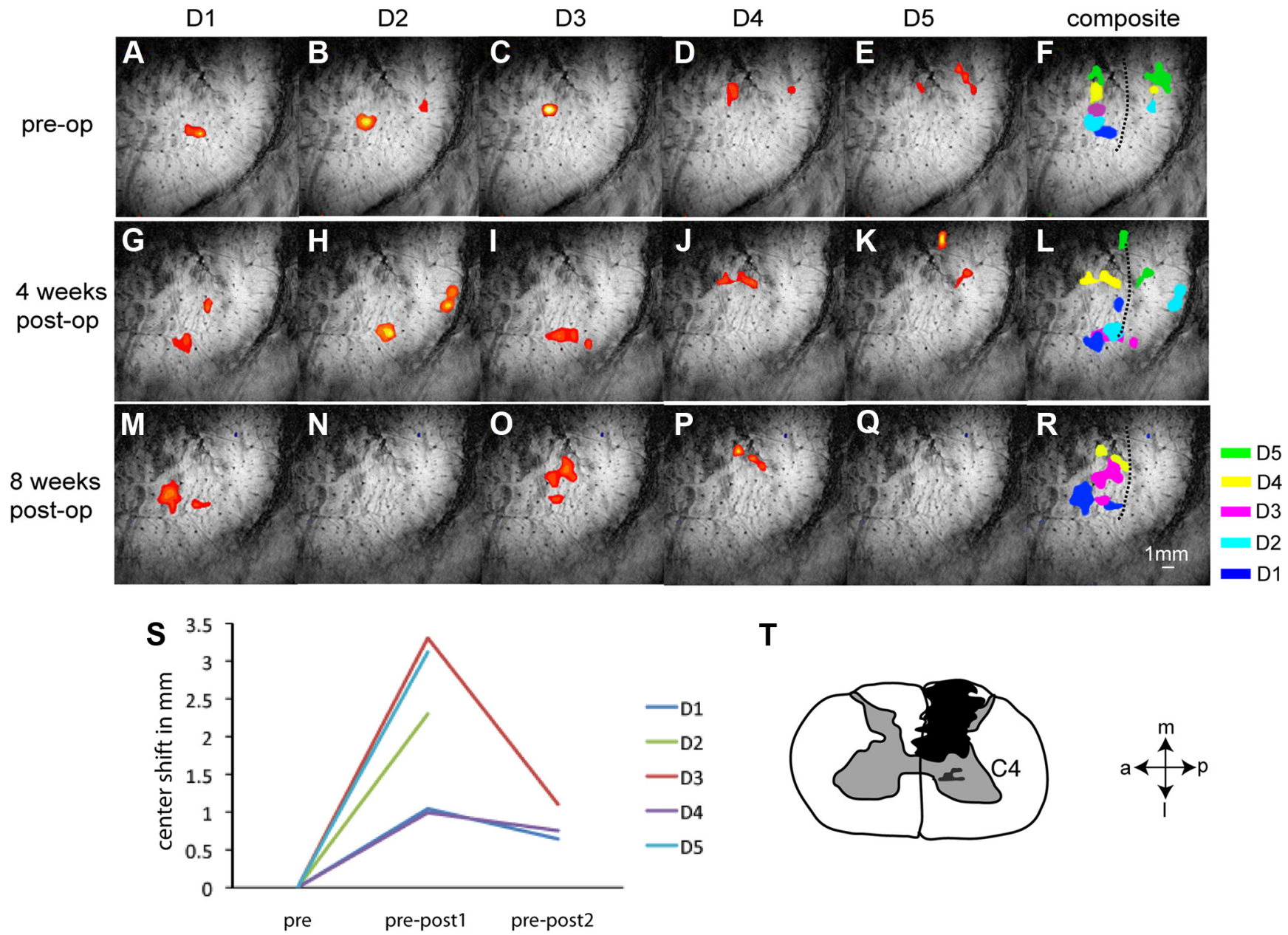

T
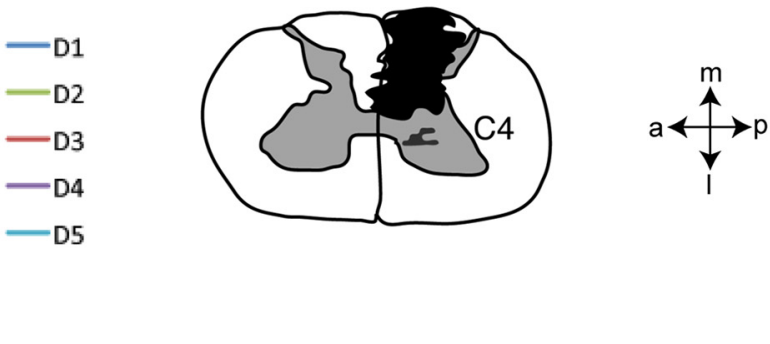

Figure 3. Longitudinal $\mathrm{fMRI}$ mapping of digit activations to tactile stimulus before and after unilateral partial dorsal column lesion in monkey $S M-0 . A-F$, fMRI activations of the individual digits D1-D5 in response to tactile stimulation before the lesion and a color-coded composite map of all five digit activations (blue, D1; light blue, D2; pink, D3; yellow, D4; green, D5). Activation maps were thresholded at $p \leq 10^{-4}$. Dotted line in $\boldsymbol{F}$ indicates the approximate border between area $3 \mathrm{~b}$ and area $1 . \mathbf{G}-\mathbf{L}$, fMRI activations of the individual digits D1-D5 and a composite map of all five digits at 4 weeks after lesion. $\boldsymbol{M}-\boldsymbol{R}$, fMRl activations of the individual digits D1-D5 and a composite map of all five digits at 8 weeks after lesion. $S$, Plot of activation center shifts (in millimeters) of each digit as a function of time after lesion. $\boldsymbol{T}$, Spinal cord reconstruction showing the level ( $(4$, cervical 4 ) and spatial extent of the lesion (black patch). Scale bar, $1 \mathrm{~mm}$. a, Anterior; I, lateral; $\mathrm{m}$, medial; p, posterior.

$3 L$, yellow patch) expanded in an anteroposterior direction, and D5 activation (green patch) shifted medially. At week 8, digit activations were only detected for three of the five digits: D1 (Fig. $3 M)$, D3 $(O)$, and $\mathrm{D} 4(P)$. D1 and D4 activations appeared to shift back slightly to their pre-lesion activation sites compared with the activations at post-lesion week 4. D3 activation showed drastic spatial shift. A plot of cortical distances between prelesion and post-lesion activation centers (Fig. 3S) as a function of time after lesion illustrated clear increases in cortical distance, indicating large, above normal $(\sim 0.5 \mathrm{~mm})$ activation center shifts for all digits at 4 weeks after lesion (pre-post1), and subsequently illustrated distance decreases, indicating the return of activation centers to their original sites at 8 weeks after lesion (D1, dark blue line; D4, purple line; and D3, red line). The centers for D1 and D4 show relatively small shifts at 4 weeks and then a small return between 4 and 8 weeks. The D3 representation, in contrast, did shift substantially in the 4-week image shift (up to $3.3 \mathrm{~mm}$; Fig. 3S, red line) and again in the 8-week image for this monkey (SM-O).

For monkey SM-D, before the lesion, $8 \mathrm{~Hz}$ tactile stimulation of individual distal finger tips evoked robust and orderly organized activations of D1-D5 in area 3b (Fig. 4A-E; Fig. 4F: D1, blue; D2, light blue; D3, violet; D4, yellow; D5, green) and of D2-D3 in area 1 (Fig. 4B,C,F). Activations of D2 and D3 in area 1 primarily overlapped (Fig. $4 F$, left side field of view), a finding that is consistent with our previous findings in normal animals (Chen et al., 2007; Friedman et al., 2008; Qi et al., 2011). After an incomplete lesion at the C5-C6 level disrupting 76\% of the afferents (Fig. 4T, as validated by histology), D1, D2, and D5 activations, but not D3 and D4 activations, were detected in the contralateral area $3 \mathrm{~b} 4$ weeks after lesion (Fig. $4 G-L$ ). In area 1, only D3 activation (Fig. 4I) was observed. The center of D5 activation shifted slightly toward the medioposterior location of the D4 middle phalange (Fig. 4L). Eight weeks after the lesion (Fig. $4 R), \mathrm{D} 1, \mathrm{D} 2, \mathrm{D} 3$, and D5 activations were detected at the same pre-lesion locations in area $3 \mathrm{~b}$ but not in area 1 (compare the left activation focus in Fig. 4C,I with that in O). D4 activations were never detected after lesion. Plots of spatial shifts (in millimeters) of individual digit centers from the pre-lesion state (defined as 0 distance) showed spatial shifts of $0.41 \mathrm{~mm}$ for D1, $0.39 \mathrm{~mm}$ for $\mathrm{D} 2$, and 0.49 for D5 at 4 weeks (pre-post1) and $0.59 \mathrm{~mm}$ for D1, $0.43 \mathrm{~mm}$ for D2, $0.49 \mathrm{~mm}$ for D3, and $0.54 \mathrm{~mm}$ for D5 at 8 weeks (pre-post2) after lesion (Fig. 4S). Except for D4, in which activation was not detected after lesion, the spatial shifts of other digits 

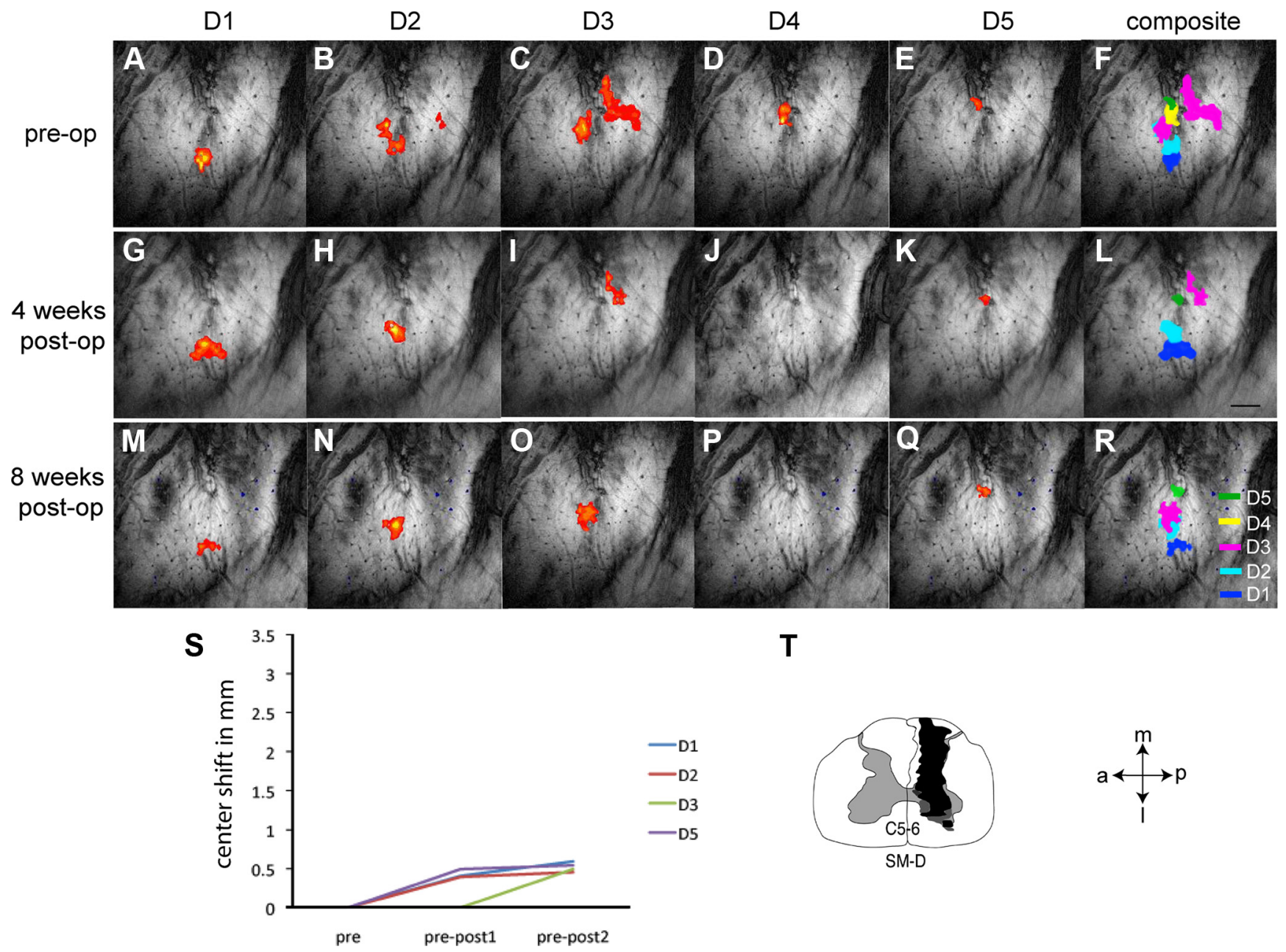

$\mathbf{T}$

Figure 4. Longitudinal fMRI mapping of digit activations to tactile stimulus before and after unilateral partial dorsal column lesion in monkey $S M-D$. $A-F$, fMRI activations of the individual digits D1-D5 in response to tactile stimulation before the lesion and a color-coded composite map of all five digit activations (blue, D1; light blue, D2; pink, D3; yellow, D4; green, D5). G-L, fMRI activations of the individual digits D1-D5 and a composite map of all five digits at 4 weeks after lesion. $\boldsymbol{M}-\boldsymbol{R}$, fMRI activations of the individual digits D1-D5 and a composite map of all five digits at 8-weeks after lesion. $S$, Plot of shifts in the activation center (in millimeters) for each digit as a function of time after lesion. $T$, Spinal cord reconstruction showing the level (C5-6) and spatial extent of the lesion (black patch). fMRI activations were thresholded at $p \leq 10^{-4}$. Scale bar, $1 \mathrm{~mm}$. a, Anterior; I, lateral; m, medial; p, posterior.

were small and comparable with those of digits with intact inputs (Zhang et al., 2010).

We observed similar activation center shifts in two other animals (SM-C and SM-F). For SM-C, two dorsal column lesions separated by 10 weeks were placed to achieve an extensive but incomplete sectioning of the afferents of the cuneate fasciculus. Combinations of these two lesions, which were placed at the $\mathrm{C} 4$ level above (rostral) the termination zones of all digit afferents and at the C4-C5 junction, disrupted $97.8 \%$ of cuneate afferents according to the quantification of tracer-labeled terminals in cuneate nuclei (for detailed histology, see Qi et al., 2011). Before lesion, fMRI revealed orderly and somatotopically organized digit activation maps in area $3 \mathrm{~b}$ (Fig. $5 A$, left) and area 1 (Fig. $5 A$, right). After the first and ineffective lesion, an orderly digit activation map was retained (Fig. $5 B$ ) at post-lesion week 5 . Activation centers of D1-D4 showed little spatial shifts $(<0.5 \mathrm{~mm})$ in relation to their pre-lesion locations (Fig. $5 A$ ). The activation center shift of D5 was the largest $(1.18 \mathrm{~mm}$; comparing green patches in $A$ and $B$, quantification in $E$ ). Similar to other cases, detection of area 1 activations was less frequent. Four weeks after the second more complete dorsal column lesion (Fig. 5C), D1 and D3 activations remained at the pre-lesion locations. D2 activation was not detected. D4 activations appeared to shift to area 3a, and D5 activation shifted medically and posteriorally. Eight weeks after the second lesion (Fig. 5D), only three digit activations were detected. The D1 activation remained at its pre-lesion site (blue patch), whereas D2 (light blue patch) and D4 (yellow patch) activations shifted to more lateral locations. D3 and D5 activations were not detected. Digit activations were mapped over 13 weeks of post-lesion recovery in SM-R because of longer behavioral testing as a result of more persistent behavioral deficits (Fig. 6). Three and 6 weeks after the lesion (only three digits were mapped in each session), fMRI activations of D1 (blue patch), D2 (light blue patch), and D3 (violet patch) shifted anteriorly and medially (Fig. 6B). D4 activation (yellow patch) shifted posteriorly and laterally. D5 activation (green patch) moved slightly toward the palm region. D4 and D5 activations in area 1 (left side patches) were also detected. Thirteen weeks after lesion, identical stimuli only evoked detectable activations for D1, D3, and D4 (Fig. 6C). D1 activation returned toward its pre-lesion location, whereas D3 and D4 activations stayed away from their pre-lesion activation sites. Plots of activation center shifts (in millimeters) as a function of post-lesion time points (Fig. $6 \mathrm{E}$ ) showed a different trend than in the other three animals. In contrast to the returning patterns of most digit activation centers in SM-C and SM-O, D3 and D4 showed increased inter-activation distance (purple and 


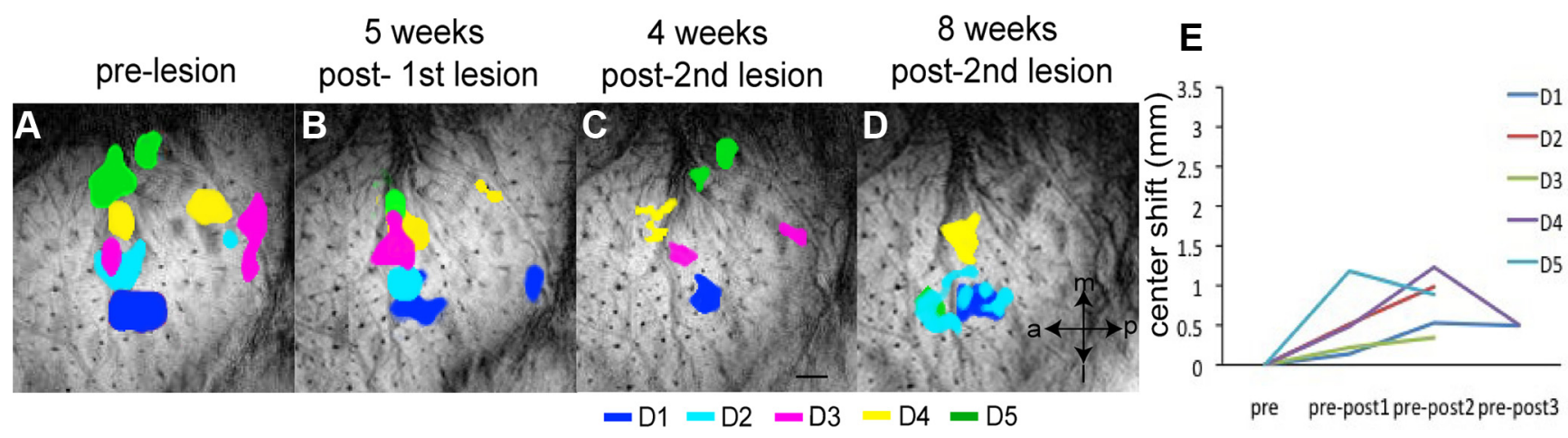

Figure 5. Longitudinal fMRI mapping of digit activations to tactile stimulus before and after unilateral partial dorsal column lesion in monkey SM-C. Composite fMRI activation maps of all five digits were obtained before $(\boldsymbol{A}), 5$ weeks after the first lesion $(\boldsymbol{B})$, and 4 weeks $(\boldsymbol{C})$ and 8 weeks $(\boldsymbol{D})$ after the second lesion. Plot of shifts in the activation center (in millimeters) for each digit as a function of time points after lesion was shown in $\boldsymbol{E}$. Scale bar, $1 \mathrm{~mm}$. a, Anterior; I, lateral; $\mathrm{m}$, medial; p, posterior.

\section{pre-les} post-les wks 3-6 post-les wks 13 fMRI-ele

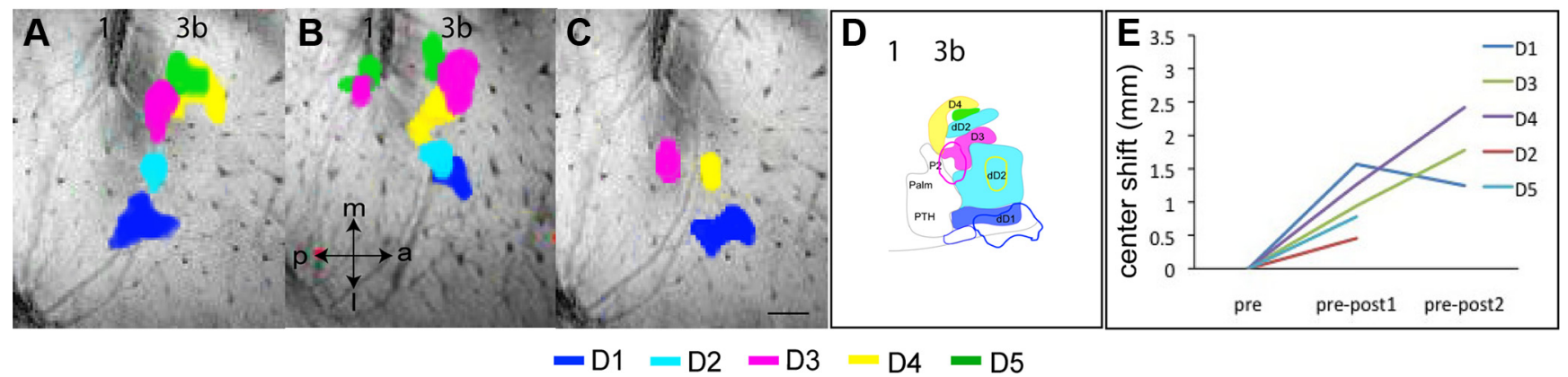

Figure 6. Longitudinal fMRI mapping of digit activations to tactile stimulus before and after unilateral partial dorsal column lesion in monkey SM-R. Composite fMRI activation maps of all five digits were obtained before $(\boldsymbol{A}), 3-6$ weeks after the lesion $(\boldsymbol{B})$, and 13 weeks after the lesion $(\boldsymbol{C})$. Composite post-lesion digit activations (color circles) at 13 weeks were overlaid on digit representation maps (solid color patches) defined by microelectrode mapping method, by characterizing multiunit spiking activity (D). Plot of shifts in the activation center (in millimeters) for each digit as a function of time points after lesion was shown in $\boldsymbol{E}$. Scale bar, $1 \mathrm{~mm}$. a, Anterior; I, lateral; m, medial; p, posterior; PTH, thenar pad.

green lines). D5 showed slightly reduced distance (blue line). Interestingly, the behavioral deficits (such as success rate and number of flexes) of this animal in food reaching and retrieving tasks remained at 13 weeks. Thus, the massive spatial reorganization of digit responses was associated with long-term behavioral deficits as a result of a nearly complete lesion. Electrophysiologically, the hand representation was somatotopically abnormal (Fig. 6D). The representation of D1 was reduced in size, with half of the D1 territory responding to the distal D2. Receptive fields on D1 and D4 were abnormally large, and there was no clear somatotopy within the digit territory. The representations of D3 and D4 were displaced medially, and the representation of D2 was abnormally large and misplaced. No site responded to D5. The thenar pad next to D1 was overrepresented without clear somatotopy. Other pads of the palm were under-represented. There was only a low degree of spatial correlation between the fMRI and electrophysiology results for D3 and D1 activations.

In summary, during the 8-13 week recovery period after a nearly complete loss of dorsal column afferent inputs from the hand, $\mathrm{fMRI}$ responses to digit stimulation remained. However, in three of the four animals (SM-C, SM-D, SM-O), locations of most of the digit activations underwent a dynamic shifting away from, and then a shifting back toward, their original pre-lesion activation sites (for summary model, see Fig. 13).

\section{Enlarged OI activations of digits after dorsal column lesions} Because longitudinal fMRI maps showed changes in digit activation locations after dorsal column lesions, we next validated and compared fMRI findings with those obtained using other highresolution mapping methods, OI, and dense microelectrode penetrations at 8 weeks after lesion. In monkey SM-O, there were clear differences between OI activations of individual digits in input-deprived contralateral cortex and those in the input-intact ipsilateral cortex (Fig. 7, compare $A-G$ with $H-K$ ). On the contralateral side, tactile stimulation of the lower lip (as a control) and input-deprived digits (Fig. 7A-F) elicited robust and somatotopically organized activations (dark regions in the images). Compared with individual digit activations of input-intact digits in the ipsilateral cortex (Fig. $7 H-K$ ), activations of inputdeprived digits were, in general, larger and less regular in shape. For example, the size of the input-deprived D1 activation (Fig. $7 B$, blue outline) was $\sim 30 \%$ greater than that of the input-intact D1 activation (Fig. $5 H$, blue outline). Lower lip activation (Fig. $7 A$, orange outline) was located laterally, as expected based on the known somatotopic organization of this region. The lower lip and D1 activations were separated by a clear inactive zone, an indication of the hand-face border (Fig. 7D). In area 1, D1 and D2 stimulation evoked two primarily overlapping activations (Fig. 7D). One small anterior activation focus evoked by $\mathrm{D} 4 \mathrm{stim}-$ ulation (Fig. 7D, yellow outline) appeared to be in area $3 \mathrm{a}$, along with the anterior tip of the D1 activation. On the ipsilateral inputintact side, digit activations were more focal $(\sim 1 \mathrm{~mm}$ in diameter) than those on the contralateral side and organized in a topographic manner (Fig. $7 K$ : D1, blue outline; D3, violet outline; D4, yellow outline). Additionally, D1 stimulation also acti- 

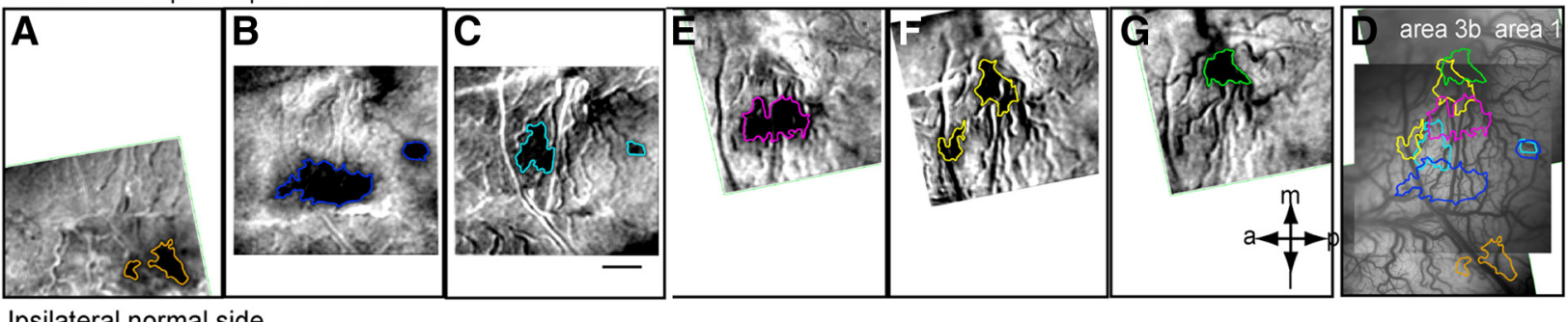

Ipsilateral normal side
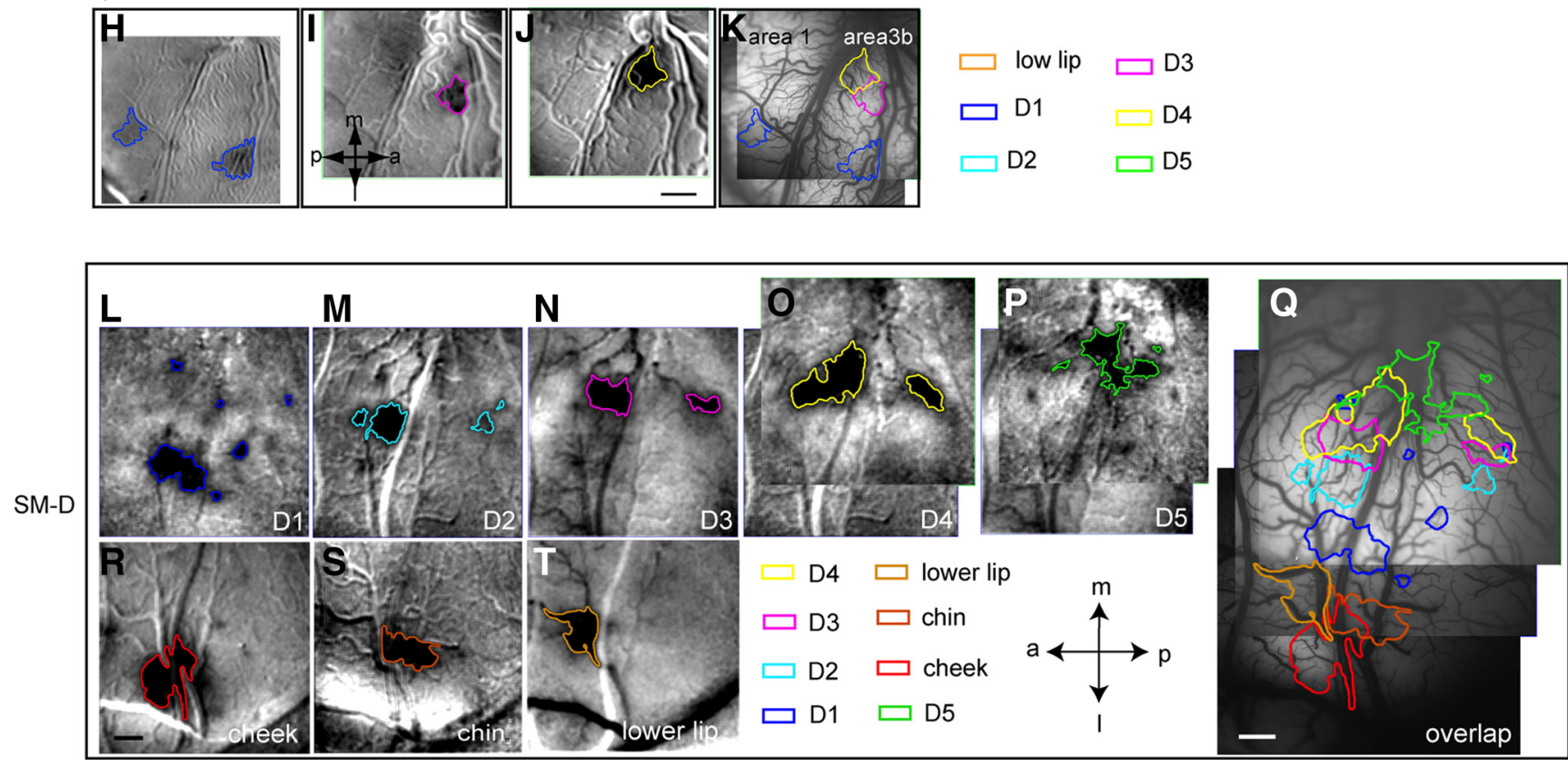

Figure 7. Ol activation maps of individual digits and facial regions of areas $3 \mathrm{~b}$ and 1 in two dorsal column lesioned monkeys (SM- 0 and SM-D). A-G, Single-condition activation maps of lower lip and D1-D5 in response to tactile stimuli in contralateral area 3 b and area 18 weeks after lesion. $\boldsymbol{D}$, Composite map of contralateral digit and face activations in SM-0. $\boldsymbol{H}-\boldsymbol{J}$, Single-condition activation maps of D1, D3, and D4 of the cortex ipsilateral to the lesion site. $\boldsymbol{K}$, Composite maps of single-digit activations in area $3 \mathrm{~b}$ and area $1 . \mathbf{L}-\boldsymbol{P}$, Single-condition activation maps of D1-D5 to tactile stimuli in contralateral area $3 \mathrm{~b}$ and area 1 in SM-D. $\boldsymbol{R}-\boldsymbol{T}$, Single-condition activation maps of cheek, chin, and lower lip in hemisphere contralateral to the lesion site. $\boldsymbol{Q}$, Composite map of digit and face activations. Scale bar, $1 \mathrm{~mm}$. a, Anterior; I, lateral; m, medial; p, posterior.

vated a smaller region in area 1 (Fig. $7 \mathrm{H}, \mathrm{K}$, blue outline on the left side).

For monkey SM-D, $8 \mathrm{~Hz}$ vibrotactile stimulation of distal finger tips of D1-D5 (Fig. 7L-P) evoked robust and focal OI activations in area $3 \mathrm{~b}$ (left side of the images) and area 1 (right side of the images) in a pattern similar to fMRI activations from these digits. Although area 3b activations for D1-D5 maintained a general lateral-to-medial somatotopy (see the composite map in Fig. 7Q), the D4 activation (yellow outline) was larger than the D1 (blue outline), D2 (light blue), or D3 (violet) activation. The digit activation sizes in area 1 were smaller than those in area $3 \mathrm{~b}$ (compared with the distance spanned from D1-D5 in area $3 \mathrm{~b}$ and in area 1); these findings were consistent with the results of our previous OI studies in normal squirrel monkeys (Chen et al., 2003, 2005; Friedman et al., 2008). Stimulation of the lower lip (Fig. $7 R-T$, dark yellow outline), chin (orange outline), or cheek (red outline) also elicited activations at somatotopically appropriate locations.

We also observed different patterns and shapes of digit activations during $\mathrm{OI}$ in contralateral and ipsilateral area $3 \mathrm{~b}$ in SM-C (Fig. 8). OI activation territories of D1-D5 were organized somatotopically in a lateral-to-medial order in the contralateral inputdeprived area $3 \mathrm{~b}$ (Fig. $8 G$ ), in an arrangement that was only somewhat different from the fMRI activation maps of digits in this animal. The D1 activation (Fig. $8 B$ ) was of normal size ( $~ 1$ $\mathrm{mm}$ in diameter); however, D2 (Fig. $8 C$ ), D3 (Fig. $8 D$ ), and D4 (Fig. $8 E$ ) activations appeared to be smaller. As a control, stimulation of the anterior chin evoked a focus of activation lateral to D1 region (Fig. $8 A, G$ ). On the ipsilateral input-intact side, activations of anterior chin (Fig. $8 \mathrm{H}$ ) to D1-D5 (Fig. $8 \mathrm{I}-\mathrm{M}$ ) exhibited regular shapes and were orderly organized (Fig. $8 N$ ). The size of single-digit activation was $\sim 1 \mathrm{~mm}$ in diameter, which is comparable with normal digit activations. The close, but not overlapping, proximity of the D1 and anterior chin activations indicate the location of the hand-face border. The majority of area 1 was out of the imaging field of view. Subsequent electrophysiological mapping confirmed the small D4 activation in area 1 (Fig. $8 \mathrm{~N}$, small yellow outline).

\section{Spatial correspondence of post-lesion fMRI, OI, and electrophysiology digit maps}

We were able to correlate fMRI and OI activations in the inputdeprived digit regions with microelectrode electrophysiology maps obtained by recording from a dense array of electrode penetrations in both input-deprived and input-intact hemispheres (Figs. 9, 10). The ipsilateral hemisphere was used as a control for cortical responsiveness and input-intact digit responses (in addition to the input-intact digit responses of normal animals). Al- 

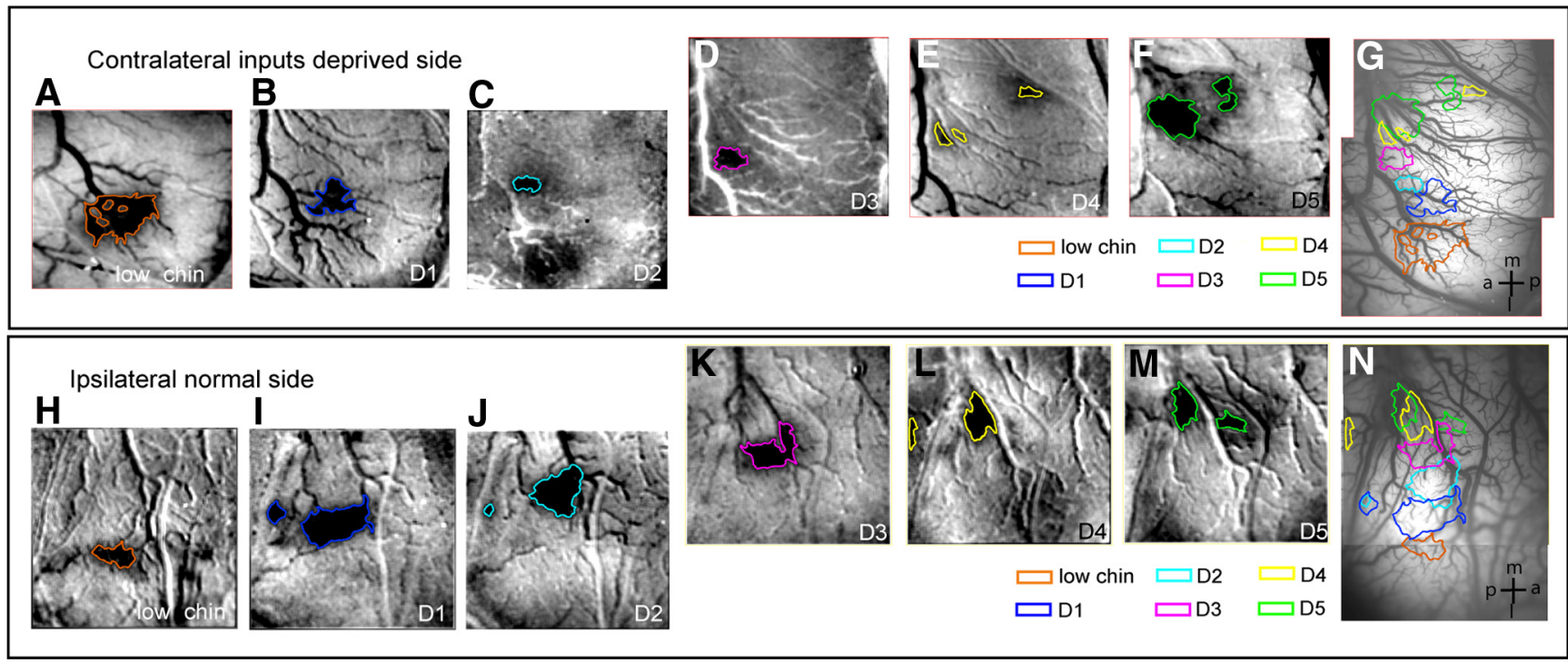

Figure 8. Ol activation maps of individual digits and facial on contralateral and ipsilateral area $3 \mathrm{~b}$ in SM-C. $A-F$, Single-condition activation maps of lower chin and D1-D5 in response to tactile stimuli in contralateral area 3b 8 weeks after the second lesion. G, Composite map of contralateral digit and face activations. $\mathbf{H}-\mathbf{M}$, Single-condition activation maps of low chin and D1-D5 in area $3 \mathrm{~b}$ ipsilateral to the lesion site. $\boldsymbol{N}$, Composite maps of individual digit activations. a, Anterior; I, lateral; $\mathrm{m}$, medial; p: posterior.
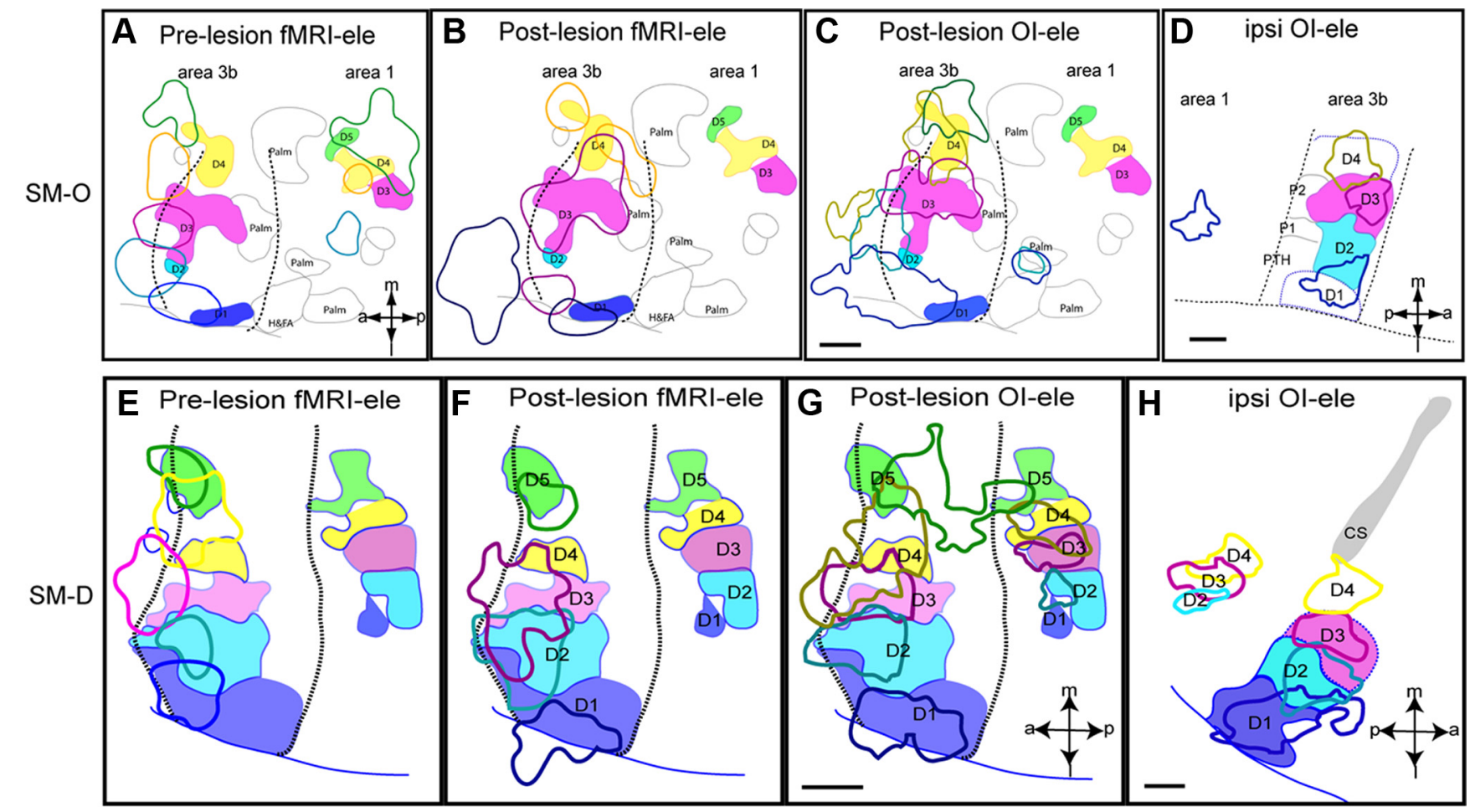

Figure 9. Spatial correspondence of fMRI, 01 , and electrophysiology maps of digit activation after dorsal column lesion in contralateral and ipsilateral areas $3 \mathrm{~b}$ and 1 in monkeys SM-0 and SM-D. $A, E$, Overlay of pre-lesion fMRI and post-lesion electrophysiology maps of digits. Color outlines indicate location and size of fMRI activations, whereas color patches present location and size of neuronal responses. $\boldsymbol{B}, \boldsymbol{F}$, Spatial comparison of post-lesion fMRI and post-lesion electrophysiology digit maps. C, G, Spatial comparison of post-lesion 0 a and post-lesion electrophysiology digit maps. $\boldsymbol{D}, \boldsymbol{H}, 0$ verlay of electrophysiology maps of digits D1-D4 in the ipsilateral area $3 \mathrm{~b}$ and area 1 of the same animals (SM-0 and SM-D). Dotted black lines, Estimated inter-areal borders. Scale bars, $1 \mathrm{~mm}$. a, Anterior; I, lateral; m, medial; p, posterior; CS, central sulcus; H\&FA, hand and forearm; PTH, thenar pad.

though the ipsilateral hemisphere is not completely normal because of the disruption of interhemispheric interactions (Reed et al., 2011), microelectrode maps of the somatotopy of area $3 \mathrm{~b}$ contralateral to deprived cortex in the present cases was not notably different from maps in intact monkeys. For monkey SM-O, much of the input-deprived digit regions in areas $3 b$ and 1 were unresponsive electrophysiologically at 8 weeks after spinal cord lesion, with islands of neurons with enlarged receptive fields and weak responses. Compared with the pre-lesion digit activation fMRI map (Fig. 9A, color outlines), there were apparent spatial shifts in the remaining digit responses toward the palm (Fig. $9 A-C$, open black outlines) and inter-area border of areas $3 \mathrm{~b}$ and 1 at 8 weeks after lesion. The remaining responsive regions of D1 (blue patch) and D2 (light blue patch) were quite small compared 


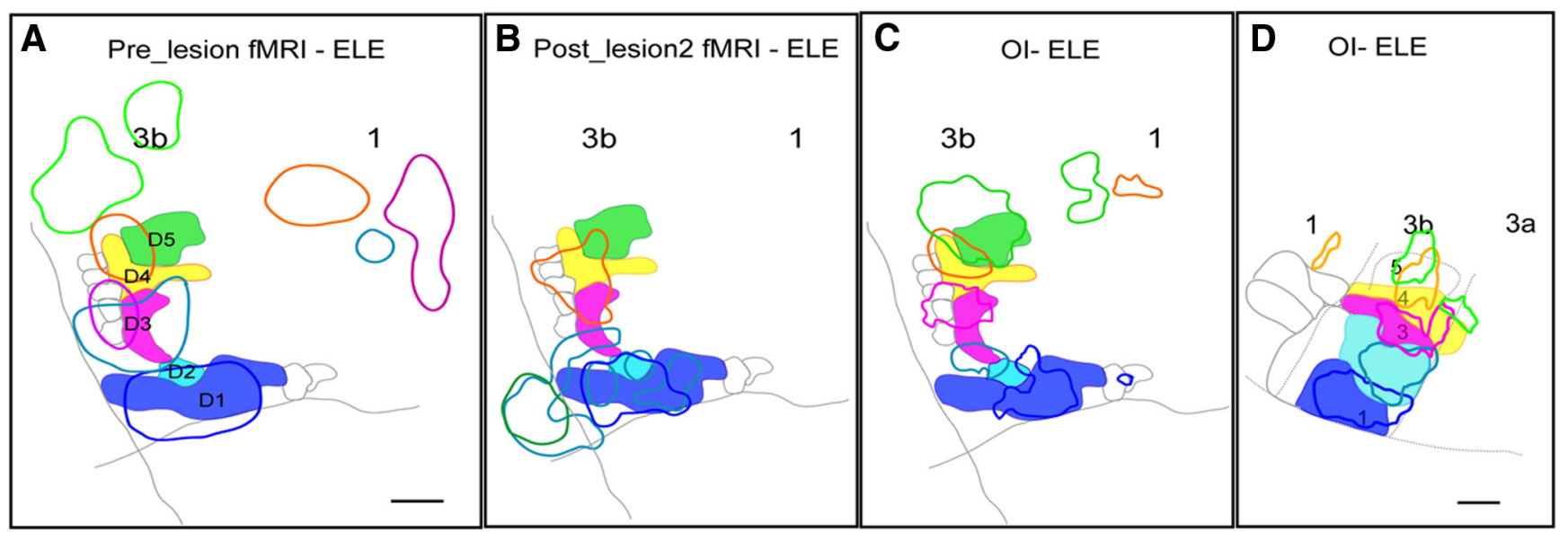

Figure 10. Spatial correspondence of $\mathrm{FMRI}, 0 \mathrm{O}$, and electrophysiology maps of digit activation before and after dorsal column lesion in contralateral and ipsilateral areas $3 \mathrm{~b}$ and 1 in $\mathrm{monkeys} \mathrm{SM}-\mathrm{C}$. $A$, Overlay of pre-lesion fMRI and post-lesion electrophysiology maps of D1-D5. Color outlines indicate location and size of fMRI activations, whereas color patches present location and size of neuronal responses. B, Spatial comparison of post-lesion fMRI and post-lesion electrophysiology digit maps. C, Spatial comparison of post-lesion 0 l and post-lesion electrophysiology digit maps. D, Overlay of 01 and electrophysiology maps of digits D1-D4 in the input normal ipsilateral area 3b and area 1 of the same animals (SM-C). Gray lines, Estimated inter-areal (area 3b and area 3a) and hand-face borders. Scale bar, $1 \mathrm{~mm}$. a, Anterior; l, lateral; $m$, medial; p, posterior.

with the responsive regions of D3 (violet patch) and D4 (yellow patch) in area $3 \mathrm{~b}$. There were large cortical regions that were unresponsive to various types of stimulation. Because of its buried location at the tip of the shallow central sulcus, we were not able to map the D5 region completely in area $3 \mathrm{~b}$ in this animal. Interestingly, D3-D5 electrophysiological maps in area 1 were organized in a fairly orderly manner (Fig. $9 A-C$, color patches on the right side). The post-lesion fMRI maps correlated better to the electrophysiologically defined digit maps than the pre-lesion fMRI map did (Fig. 9, compare $A, B$ ). At 8 weeks after lesion, D1 (dark blue outline), D3 (dark red outline), and D4 (orange outline) fMRI activations were located at somatotopically appropriate sites, but the sizes of the activation sites were larger than normal (particularly for D4; Fig. 9B). D3 stimulation evoked an fMRI response in a region that was unresponsive in the electrophysiologically defined map (Fig. 9B, red outline next to dark blue outline). This region responded to D2 stimulation in the pre-lesion fMRI map. D1 stimulation also activated a noticeably large region in area 3a (Fig. 9B, dark blue outline in the left corner). Unlike the post-lesion fMRI digit maps, the post-lesion OI maps of digits exhibited close spatial correlations with the post-lesion digit maps defined electrophysiologically (Fig. 9C). All the digit activations (D1-D5) were detected, and they were large in size (compare color outlines with color patches). The most significant size differences were present for D1 and D2 (Fig. 9C). Electrophysiology and OI maps of D3 and D4 were in close agreement (Fig. 6C). Portions of the D1 and D2 activations, as well as the second patch of D4 activation, were determined to be in area $3 \mathrm{~b}$, as indicated by the border between areas $3 \mathrm{~b}$ and $3 \mathrm{a}$ (Fig. $9 A-C$, dotted lines). In strong contrast to the contralateral input-deprived cortex, there was a very good spatial correlation between $\mathrm{D} 3 \mathrm{OI}$ and electrophysiological maps in the input-intact cortex (Fig. 9D, violet outline and patch). Activations were within the area $3 \mathrm{~b}$ region (border lines were indicated by dotted black lines). OI activations of D1 and D4 were located in somatotopically appropriate locations.

For SM-D, neurons within the D1 and D2 territories (Fig. $9 E-G$, dark blue and light blue patches) appeared normal with respect to the receptive field size and strong response strengths to tactile stimuli (details are described by Qi et al., 2011). These normal neuronal response properties suggested that the lesion primarily spared the inputs of D1 and D2; subsequent tracer tracking histological results actually confirmed the sparing of D1 afferents in this animal (Qi et al., 2011, their Fig. 3). The lesion was placed at the level of D2 afferent inputs; however, the quantity of remaining fibers of D2 was unknown, because tracer was not injected in this digit. The large, unresponsive cortical regions of D3-D5, as indicated by small violet, yellow, and green patches of D3-D5 in Figure 9E-G, match the lesion location. The remaining responsive regions contained neurons that responded weakly to digit stimulation. Using the electrophysiology map as the "ground-truth" reference, fMRI activations of D1-D3 and D5 at 8 weeks after lesion (Fig. 9E, matching color outlines) appeared to be located at topographically appropriate cortical regions, except that the D3 activation (violet outline) was unusually large. Compared with fMRI activations, OI and electrophysiology maps of D1 and D2 (spared digits) in area $3 \mathrm{~b}$ were in good spatial agreement (Fig. 9G). OI activations of input-deprived digits (D3 to D5), however, were significantly larger than the electrophysiologically defined responsive regions (Fig. 9G, compare the yellow outline of D4 OI activation and the yellow patch of the D4 electrophysiological map). Interestingly, we detected OI activations of D2-D5 in area 1 that were in general agreement with their corresponding electrophysiologically defined regions. On the ipsilateral side in which inputs were intact, OI and electrophysiology maps of D1-D3 showed close spatial agreements (Fig. 9H).

For SM-C, a fairly large portion of area $3 \mathrm{~b}$ retained electrophysiological responsiveness (color patches in $A-C$ ) to digits stimulation 8 weeks after a nearly complete lesion $(97.8 \%$ afferent disruption; Fig. 10). The post-lesion fMRI responses of D1 (Fig. $10 \mathrm{~B}$, blue outline) and $\mathrm{D} 4$ (orange outline) were in general agreement with the remaining post-lesion responsive territories defined electrophysiologically (Fig. $10 \mathrm{~B}$, color patches). For D2, multiple activation foci were detected, with one that colocalized with the electrophysiologically responsive site (Fig. $10 \mathrm{~B}$, compare light blue outlines with patch). For D5, no apparent fMRI activation was detected in area $3 \mathrm{~b}$, but an activation focus was in area $3 \mathrm{a}$ at a location anterior to the D1 territory of area $3 \mathrm{~b}$ (dark green outline anterior to the area $3 \mathrm{a}-3 \mathrm{~b}$ border). In general, post-lesion fMRI digit activation maps in area $3 \mathrm{~b}$ showed reasonable spatial correlation with electrophysiological maps of the digits. fMRI and electrophysiology activations of D1 (blue outline and patch), 

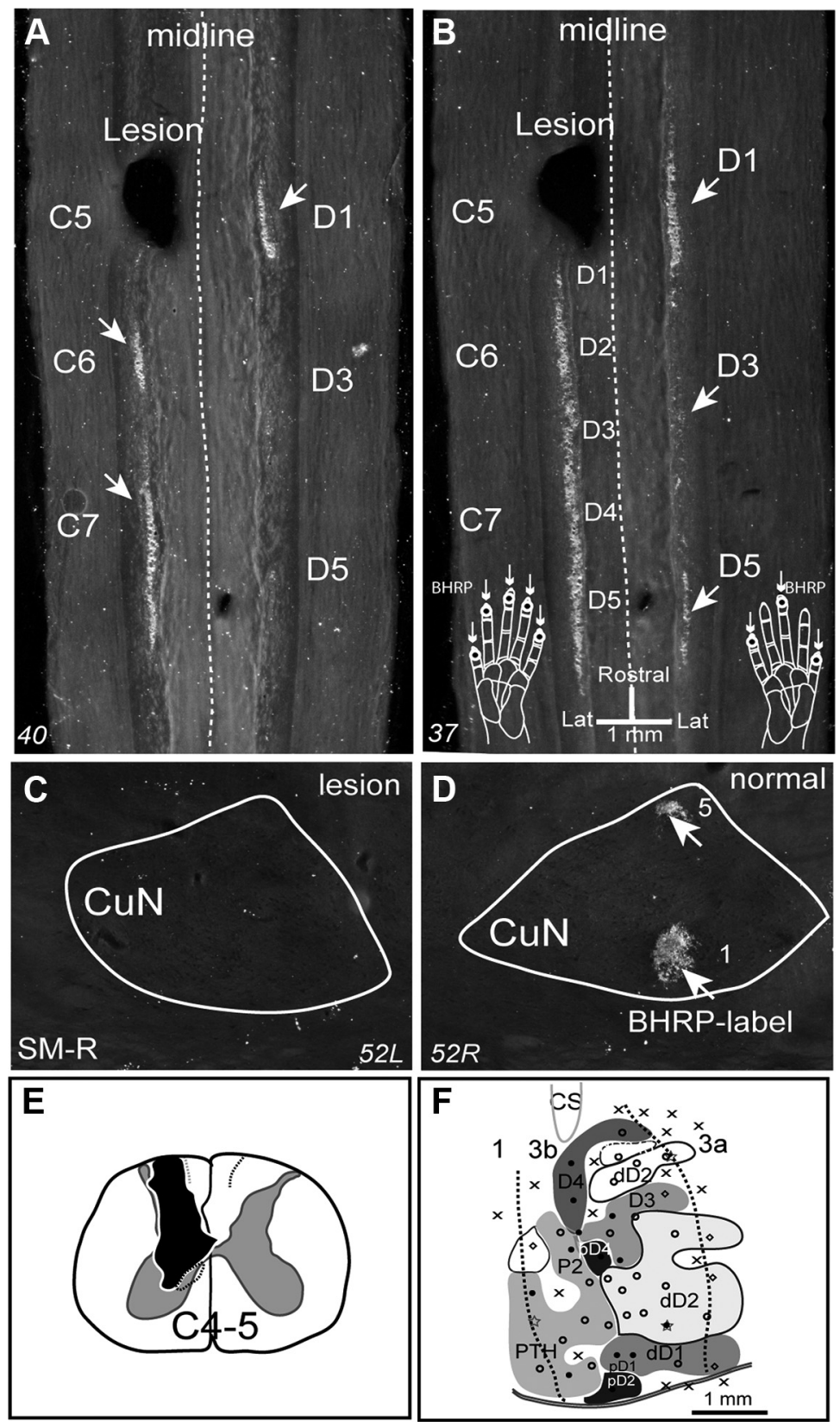

Figure 11. Photomicrographs of the cutaneous afferent terminations revealed with tracer injections of cholera toxin subunit B conjugated with wheat germ agglutinin horseradish peroxide (BHRP) in the dorsal horn of the spinal cord $(\boldsymbol{A}, \boldsymbol{B})$ and cuneate nucleus of the brainstem $(\boldsymbol{C}, \boldsymbol{D})$ and electrophysiological maps of digit representation in monkey SM-R. $\boldsymbol{A}-\boldsymbol{D}$ shows labeled terminations of tracer cholera toxin subunit $B$ conjugated with wheat germ agglutinin horseradish peroxide (BHRP) when the distal phalanges of digits 1,3 , and 5 on right hand and distal phalanges of D1-D5 on the lefthand of a squirrel monkey were injected with tracer. The cuneate nucleus is outlined on both sides of one brainstem section, and foci of terminal label are marked with white arrows. Note that there are two patches of label in the normal side of cuneate nucleus that are not present in the lesion side. A schematic drawing shows the reconstructed transverse view of spinal cord through cervical segment $(4-C 5$ indicating the extent of lesion (black shade) on the left side of the spinal cord ( $\boldsymbol{E}$ ). The lesion was reconstructed from a series horizontally cut sections. Dense microelectrode mapping and recording map shows the representations regions for individual digits $(\boldsymbol{F})$. Solid dots mark penetrations with good responses; black open circles mark those with weak responses. $3 \mathrm{~b}$, Area $3 \mathrm{~b}$; 3a, area 3a; 1, area 1; C4-C5, spinal cord cervical segment 4-5; cs, central sulcus; CuN, cuneate nucleus of the brainstem; D1-D5, digits 1-5; dD1-dD2, distal D1-D2; Lat, lateral; pD1-pD4, proximal D1-D4; P2, palm pad 2; PTH, thenar pad. Squirrel monkey (SM-R) received a unilateral dorsal column lesion that involved most if not all of the cuneate fasciculus at the 5 level but with limited damage to the gracile fasciculus and other adjoining parts of the spinal cord. The reconstructed lesion map indicated that the damage involved almost the entire cuneate fasciculus and was placed at the D1 level.

D2 (light blue outline and patch), and D4 (orange outline and yellow patch) showed a larger degree of overlap than D5. However, in area 3a (bottom left corner of the images), D2 and D5 activations overlapped (anterior side of area $3 \mathrm{a}-3 \mathrm{~b}$ border, verti- cal lines $A-C)$. Compared with the postlesion fMRI activation map (Fig. $10 \mathrm{~B}$ ), OI maps of digits had a higher spatial correspondence with electrophysiological maps (Fig. 10C). Contrary to the failed detection of fMRI responses to D3 and D5 stimulation, OI revealed D3 (Fig. 10C, violet outline) and D5 (green outline) activations in area $3 \mathrm{~b}$. Additional area 1 activations were also detected for D4 and D5. Although there were OI responses in the input-deprived digit regions, the sizes and spatial organization of the activated zones was remarkably different from the digit response zones in the input-intact ipsilateral hemisphere (Fig. 10, compare $C, D)$. In input-intact area $3 \mathrm{~b}$, OI revealed normally organized D1-D4 activations (Fig. 10D, color outlines). These activations spatially correlated quite well with digit maps defined electrophysiologically (Fig. $10 D$, color patches). Figure 11 illustrates the spatial extent and level of unilateral dorsal column lesion in SM-R and the transported tracer from digits to the spinal cord and cuneate nucleus. The tracer used in this animal differs from that used in the other three animals (SM-O, SM-D, and SM-C; Qi et al., 2011).

To summarize, although electrophysiological mapping revealed only a few cortical islands containing weakly responsive neurons in area $3 \mathrm{~b}$ after near-complete dorsal column lesions, ranging from sparing $14 \%$ to $1 \%$ of digit afferents to the cuneate nucleus, large and robust fMRI and OI activations were obtained from area 3b. OI maps of digits had a closer spatial correlation with the electrophysiology maps than with fMRI maps at 8 weeks after lesion.

\section{Changes in single-digit activation sizes} after dorsal column lesion

To determine whether digit activation sizes enlarged after spinal cord lesions, when measured by fMRI or OI, we pooled measures from all digits from deprived cortex and intact cortex (Fig. 12B-E). Digit activation sizes were larger in deprived cortex than in intact cortex when measured with OI $(t$ test, $p<0.01)$. Although the fMRI results exhibited a similar trend, the activation size increase in the input-deprived cortex was not statistically significant $(p=0.16)$ because of the greater variation in activation sizes across cases (note the large error bars in Fig. 12). Every case of deprived area $3 \mathrm{~b}$ had an abnormal fMRI map. Compared with the digit representation sizes defined by microelectrode mapping, we found significant enlargements of digit activation sizes defined functionally by fMRI $(p<0.001, n=12$, Student's $t$ test $)$ and OI $(p<0.01, n=14)$ in 

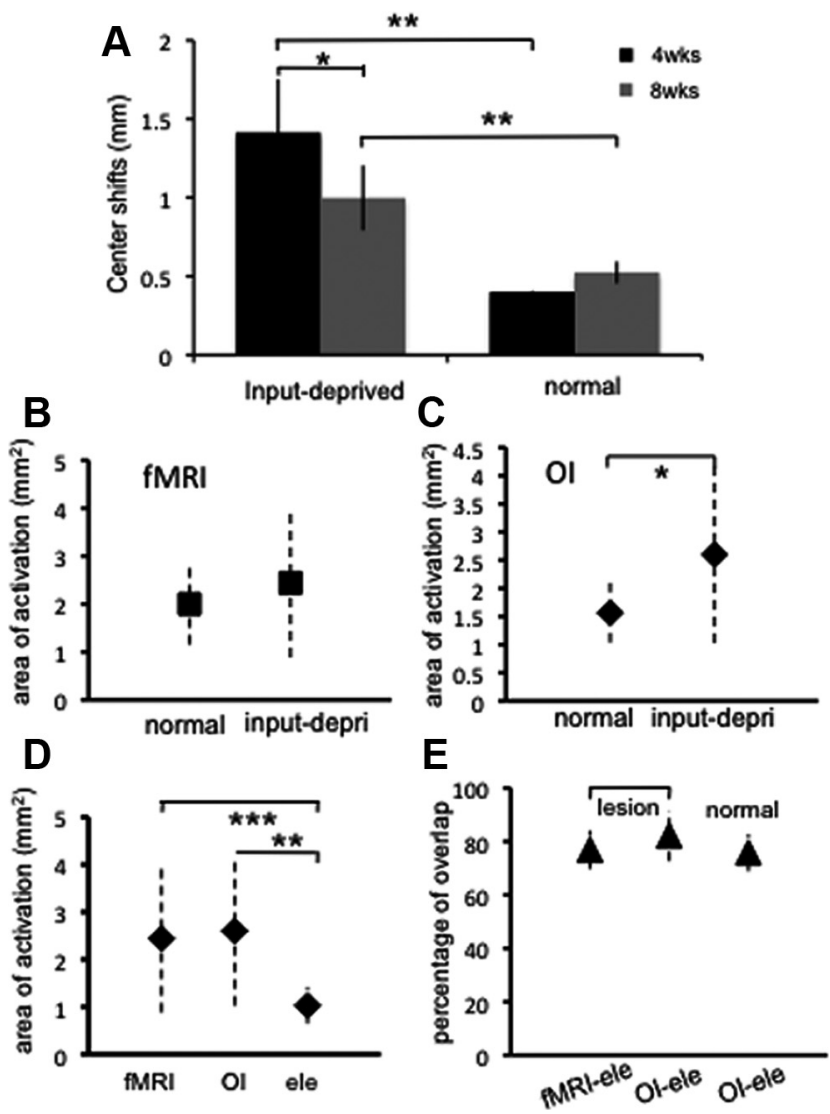

Figure 12. Center shifts of FMRI digit activations and size comparisons of single-digit activations as determined by fMRI, 01 , and electrophysiology, in input-deprived versus input-intact regions of area $3 b . A$, Plots of $f M R I$ activation center shifts (mean \pm SE) of individual digits from their pre-lesion activation sites in area $3 \mathrm{~b}$ of dorsal column lesioned animals (input-deprived) and normal animals (input-intact). Measures of individual digits of four lesioned animals (SM-D, SM-C, SM-0, and SM-R) and four normal animals (including SM-M and SM-F) are included in the calculation. $\boldsymbol{B}$, Plots of mean fMRI activation size of normal input-intact digits $(n=10)$ and input-deprived digits $(n=12)$. C, Plots of mean 0 l activation size of normal input-intact $(n=12)$ and input-deprived $(n=14)$ digits. $\boldsymbol{D}$, Comparison of mean sizes of single-digit activations of $\mathrm{FMRI}, \mathrm{Ol}$, and electrophysiology in area $3 \mathrm{~b}$ of input-deprived cortex. Error bars in $B-D$ indicate SD. $\boldsymbol{E}$, Percentages of activation area overlap between fMRI and electrophysiology (left column), 0 l and electrophysiology (middle column) in input-deprived cortex, or 0 and electrophysiology (right column) in input-intact cortex of area 3b. Error bars indicate the SE. ${ }^{*} p \leq 0.05 ;{ }^{* *} p \leq 0.01 ;{ }^{* * *} p \leq 0.001$.

input-deprived digit regions (Fig. 12D). Across all digits, the spatial agreement between OI and electrophysiology maps was slightly higher $(82.1 \pm 9.5 \%$ overlap, mean \pm SE, $n=15$; Fig. $12 E$, left column) than that between fMRI and electrophysiology (76.9 $\pm 7.1 \%$ overlap, $n=12$; Fig. $12 \mathrm{E}$, middle column) in lesioned animals. The overlap of the OI and electrophysiology maps of input-intact digits was $\sim 75.8 \pm 6.9 \%(n=14$; Fig. $12 E$, right column). Thus, the spatial overlap between functional and electrophysiological maps in input-deprived and input-intact cortex is similar. This supports the conclusion that highresolution $\mathrm{fMRI}$ and $\mathrm{OI}$ are reliable tools that can be used to map plastic changes in cortex in physiological and pathophysiological conditions.

In summary, the present study mapped longitudinally the area $3 \mathrm{~b}$ digit responsiveness to tactile stimulation before and after unilateral dorsal column lesion with fMRI and quantitatively compared the spatial correspondence of digit activation maps measured by fMRI, OI, and electrophysiology at 8 weeks or more after lesion. Although longitudinal maps of normal input digits remained stable, we obtained several significant findings in lesioned animals. First, after dorsal column lesions that disrupted $76-99 \%$ of the afferents, input-deprived digit regions remained responsive to tactile stimulation at weeks 4,8 , and up to 14 weeks after unilateral dorsal column lesion. These responses were detected by functional imaging signals (fMRI and OI) as well as by neuronal electrophysiological signals. Second, by using prelesion fMRI activation maps as a reference, longitudinal fMRI revealed a dynamic spatial reorganization of digit activations occurring between 1 and 3 months after lesion. Although there were various degrees of variation in the spatial shifts of digit activation centers, there was a general trend for digit activations to be shifted away at 4 weeks after lesion and then to return to (or close to) their original pre-lesion cortical sites at $8-13$ weeks after lesion. This type of large activation center shift was only present in input-deprived cortex and was never observed in input-intact cortex. Although the activation patterns varied across the cases of different lesion sizes and levels of reactivation, the trend of mislocated activations followed by the reestablishment of a more normal pattern was statistically significant (Fig. 12A). A model illustrates this dynamic spatial shifting of digit representations over an 8 week post-lesion period (Fig. 13, left three columns). Third, the single-digit fMRI and OI activations of the inputdeprived cortex were larger in size than those of digit activation from input-intact cortex, and single-digit activations revealed by functional signals (FMRI and OI) were significantly larger than the single-digit representation region defined by neuronal response properties (Fig. 13, right column). This indicates that there are larger regions of subthreshold activations that are not revealed by neuronal spike recordings. Finally, as a reliability measure of spatial activation size quantification, we observed no significant differences in spatial correlation between functional imaging maps of fMRI and OI or electrophysiological maps of digits, in either input-intact or input-deprived cortex. There was an $\sim 80 \%$ overlap between the functional imaging and electrophysiological maps.

\section{Discussion}

Dynamic changes in post-lesion digit activation maps and their functional implications

Because of the significantly enhanced signal or contrast to noise ratio at ultra-high MRI field strengths (e.g., 9.4 T in this study), this study along with others has demonstrated the sensitivity and reliability of high-field fMRI in revealing the fine-scale functional organization of the somatosensory system in monkeys (Chen et al., 2007; Zhang et al., 2010). With the assistance of a high precision alignment algorithm (Lecoeur et al., 2011) and rich surface blood vessel information (Zhang et al., 2010), longitudinal fMRI studies have been able to repeatedly generate stable activation maps of individual digits with a localization variation of $\sim 0.5$ $\mathrm{mm}$, the size of one voxel. This new technical advancement empowered us to study cortical functional organization at a submillimeter scale and its changes over time after spinal cord injury.

In the present study, we observed dynamic changes in digit tactile activation locations in area $3 \mathrm{~b}$ within a $2-3$ month period after unilateral dorsal column sections at high cervical spinal cord level. Although the neuronal response properties characterized at the end of the 2-3 months recovery period are similar to previous findings (Jain et al., 1997, 1998, 2008), we identified here a dynamic shift of individual digit activation centers during this recovery period. We studied the functional reorganization across a 2-3 month period because of two observations. First, our previous study showed that, in this particular partial unilateral dorsal 


\section{pre-lesion}

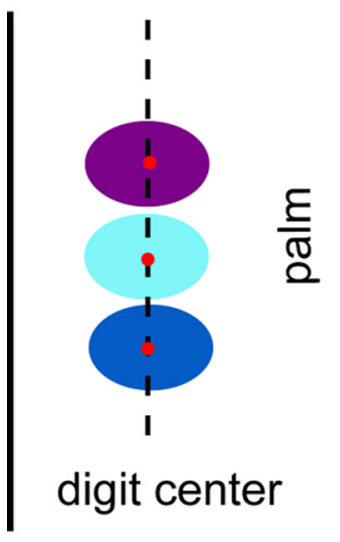

4wks post

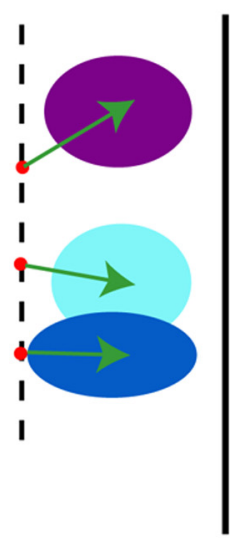

8wks post

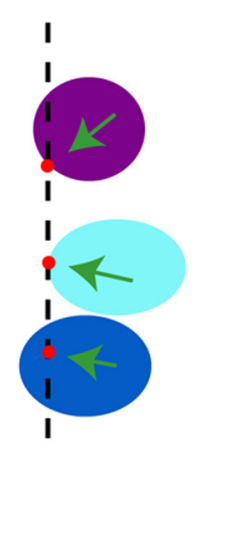

comparison

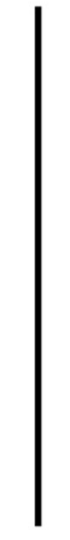

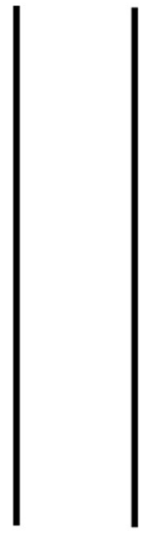

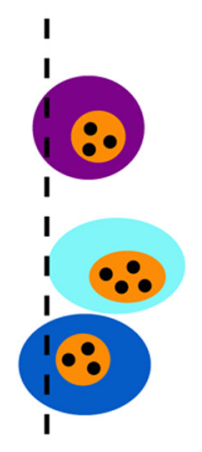

electrophysiologically defined digit response

Figure 13. Schematic summary of dynamic functional reorganization of digit representations in area $3 \mathrm{~b}$ after dorsal column section. Different color patches indicate the locations and relative sizes of fMRI activations to tactile stimulation of distal finger tips in pre-lesion (left column), 4 weeks after lesion (left middle column), and 8 weeks after lesion (right middle column). Red dots indicate the centers of pre-lesion digit activation centers. Dotted black lines indicate the center line of digit activations before lesion. Arrows represent the trends of activation shifts. Orange patches (right column) show the digit representation regions identified with neuronal firing properties. Black dots indicate the microelectrode penetration sites.

column lesion model, reorganization of digit representations tends to be stable after 2 months (Jain et al., 1997). Second, our parallel longitudinal behavioral study showed that the behavioral performance of these lesioned animals on the food reach-andretrieval task typically recovered to a pre-lesion level 2 to 3 months after the lesion (data not shown). During this period of recovery, we report two characteristic changes in cortical responsiveness to tactile stimuli of the digits as measured with fMRI: a spatial shift of digit activation centers and a spatial expansion of the responsive region. Digit activation in deprived cortex passed through a movement away phase at 4 weeks after lesion and a return phase toward its original pre-lesion cortical sites at 8-13 weeks after lesion. Because the same digits and sites on the hand were stimulated before and after dorsal column lesion, we believe the activation loci shifts reflect the time course of the reactivation of the input-deprived digit regions. The time course of these observed activation center shifts appeared to be in parallel with the time course of neuronal responsiveness changes observed using dense microelectrode mapping and recording methods (Jain et al., 1997, 1998; Qi et al., 2011) as well as the recovery of behavioral performance on the food reach-and-retrieval task. The reasons for the local spatial shift remain to be determined. It is possible that the continued use of a sensory impaired hand drives the recovery of a more normal somatotopic map. In addition, because fMRI signals reflect activities originating from large populations of neurons, the response center shifts could indicate a local spatial response profile change in the responsiveness of different adjacent digit regions.

The second important finding of this study is the enlarged cortical responsive areas (as indicated by greater single-digit activation size) of input-deprived digits to tactile stimuli 8 or 13 weeks after lesion. Based on a proposed two-phase model of the reorganization (Garraghty et al., 1991, 2006), this enlargement response noted in the late phase could be mediated by synaptic and intracortical connection changes and could be a reflection of local disinhibition during recovery after the cortex loses its peripheral inputs (Garraghty et al., 2006). Although the pooled population data from all the digits supports the trend of increase in digit activation sizes, there were apparently large variations in actual activation size. We attribute this large variation to differences in the degree of changes of different digits attributable to their specific relationship to the completeness and size of the cortical deactivation zone and cortical distance from cortex that remained responsive, which varied from 1 to $3 \mathrm{~mm}$ in cortical distance. Although the small number of input deprivation samples (four cases) collected in this study does not allow us to quantify the cross-digit and cortical-distance-dependent variations of digit responsiveness, there was evidence suggesting that the cervical level of the lesion (in which digits were spared) and the extent of the remaining inputs within the digit representation regions affected the reactivation of the deprived cortical regions (Florence et al., 1996; Jain et al., 1997; Qi et al., 2011). Although enlarged cortical response regions to tactile stimuli could be attributed to many possible factors, we can rule out at least one possibility: that of signal differences between our measuring methods of fMRI and OI. When we used electrophysiologically defined digit maps as the gold-standard reference, the spatial correspondence (as indicated by $\sim 80 \%$ overlap of the activation region) between the fMRI and OI maps with the electrophysiology maps was quite comparable, regardless of the status of the cortex: input-deprived or input-intact (Fig. 7).

\section{Similarities and differences among digit maps revealed by different methods}

With the goal of fine-scale longitudinal mapping of cortical plasticity in mind, it is essential to understand quantitatively the spatial correspondences between functional maps (both fMRI and OI) and electrophysiological maps. From a methodological point of view, functional signals can be evaluated from at least two perspectives: response detectability and localization precision. There is no dispute that neuronal electrophysiology provides the richest and most detailed information about the functional organization of a region and changes in neuronal response properties (e.g., receptive field size and response magnitude). Because the microelectrode-mapping method is labor intensive and time consuming, noninvasive (fMRI) and relatively noninvasive (OI) functional imaging offer more rapid mapping methods for monitoring brain plasticity and ultimately for correlating cortical ac- 
tivation with behavioral performance (Frostig, 2006; Frostig et al., 2008). For instance, a complete set of digit maps in multiple cortical areas can be acquired within hours, whereas obtaining the same set of electrophysiology data would require a couple of days. Compared with electrophysiology maps of digits, OI signals offer high sensitivity and high spatial precision (because of its super spatial resolution of $\sim 100 \mu \mathrm{m}$ ) in detecting stimulus-related activations in all digits in multiple cortical areas (Bonhoeffer and Grinvald, 1991; Grinvald et al., 1986), in both input-deprived and input-intact digit regions. In contrast, detectability of fMRI signals was lower than OI signals, as evidenced by undetected digit activations, which in contrast were detectable by OI and electrophysiology. Nevertheless, once detected, the spatial correspondence among fMRI and OI and electrophysiology maps were all quite good. Here we provide evidence that both high-field fMRI and OI have great potential for quantitatively monitoring cortical plasticity and reactivation.

\section{Possible roles of subthreshold electrical activation in input-deprived cortex}

In this experimental model, OI and fMRI activations were quite robust in digit regions in which inputs were severely disrupted, and neurons showed only very weak spiking activity (for a detailed description of the neuronal responsiveness, see Qi et al., 2011). This discrepancy between robust functional imaging signals and weak spiking activity led us to speculate that significant amounts of non-spiking neuronal activity exist in the inputdeprived cortex. Thus, the locations and extents of sensory activations are underestimated if only spiking activity is measured. Because it is known that functional imaging signals, including fMRI, BOLD, and OI of intrinsic signals, capture a large portion of the signals related to subthreshold synaptic activity in addition to spiking activities, the enlarged responsive region likely reflects a substantial level of subthreshold electrical activity in the input-deprived cortex that is missed by spiking measures. Those subthreshold synaptic activities could be essential for the reemergence of spiking activity by the neurons in the region and might play an important role in the functional recovery of the region. The immunohistochemical results of Garraghty and colleagues suggest that reduced inhibition in the deprived cortex contributes to the elevated subthreshold synaptic activity. During the recovery period, synaptic activities may be ramped up to levels that restore the spiking activities (Garraghty et al., 2006; Mowery and Garraghty, 2009). Our observations suggest that subthreshold activity plays an important role in the reactivation of deafferented cortex.

In conclusion, the results indicate that high-resolution fMRI can be used to effectively monitor the course of cortical reactivation after sensory loss attributable to spinal cord injury. More importantly, the fMRI results reveal dynamic changes in the cortical activation pattern during cortical recoveries that were previously unknown and correlate well with behavioral recovery. Potentially, similar fMRI scans in humans can be used to predict levels of behavioral recovery and guide behavioral and other treatments. Finally, the optical and fMRI imaging results reveal activations that are subthreshold for neural spiking and are not apparent in the microelectrode maps but may be potentiated to promote behavioral recovery.

\section{References}

Almli CR, Finger S (1988) Toward a definition of recovery of function. New York: Plenum.

Bonhoeffer T, Grinvald A (1991) Iso-orientation domains in cat visual cortex are arranged in pinwheel-like patterns. Nature 353:429-431.
Buonomano DV, Merzenich MM (1998) Cortical plasticity: from synapses to maps. Annu Rev Neurosci 21:149-186.

Chen LM, Friedman RM, Ramsden BM, LaMotte RH, Roe AW (2001) Finescale organization of SI (area 3b) in the squirrel monkey revealed with intrinsic optical imaging. J Neurophysiol 86:3011-3029.

Chen LM, Heider B, Williams GV, Healy FL, Ramsden BM, Roe AW (2002) A chamber and artificial dura method for long-term optical imaging in the monkey. J Neurosci Methods 113:41-49.

Chen LM, Friedman RM, Roe AW (2003) Optical imaging of a tactile illusion in area $3 \mathrm{~b}$ of the primary somatosensory cortex. Science 302:881-885.

Chen LM, Friedman RM, Roe AW (2005) Optical imaging of SI topography in anesthetized and awake squirrel monkeys. J Neurosci 25:7648-7659.

Chen LM, Turner GH, Friedman RM, Zhang N, Gore JC, Roe AW, Avison MJ (2007) High-resolution maps of real and illusory tactile activation in primary somatosensory cortex in individual monkeys with functional magnetic resonance imaging and optical imaging. J Neurosci 27:9181-9191.

Chen LM, Friedman RM, Roe AW (2009) Area-specific representation of mechanical nociceptive stimuli within SI cortex of squirrel monkeys. Pain 141:258-268.

Chen LM, Dillenburger BC, Wang F, Friedman RM, Avison MJ (2011) High-resolution functional magnetic resonance imaging mapping of noxious heat and tactile activations along the central sulcus in New World monkeys. Pain 152:522-532.

Cooper BY, Glendinning DS, Vierck CJ Jr (1993) Finger movement deficits in the stumptail macaque following lesions of the fasciculus cuneatus. Somatosens Mot Res 10:17-29.

Darian-Smith C, Ciferri M (2006) Cuneate nucleus reorganization following cervical dorsal rhizotomy in the macaque monkey: its role in the recovery of manual dexterity. J Comp Neurol 498:552-565.

Darian-Smith C, Ciferri MM (2005) Loss and recovery of voluntary hand movements in the macaque following a cervical dorsal rhizotomy. J Comp Neurol 491:27-45.

Duong TQ, Kim DS, Uğurbil K, Kim SG (2001) Localized cerebral blood flow response at submillimeter columnar resolution. Proc Natl Acad Sci U S A 98:10904-10909.

Finger S, Almli CR (1985) Brain damage and neuroplasticity: mechanisms of recovery or development? Brain Res 357:177-186.

Flor H, Elbert T, Knecht S, Wienbruch C, Pantev C, Birbaumer N, Larbig W, Taub E (1995) Phantom-limb pain as a perceptual correlate of cortical reorganization following arm amputation. Nature 375:482-484.

Flor H, Braun C, Elbert T, Birbaumer N (1997) Extensive reorganization of primary somatosensory cortex in chronic back pain patients. Neurosci Lett 224:5-8.

Florence SL, Jain N, Pospichal MW, Beck PD, Sly DL, Kaas JH (1996) Central reorganization of sensory pathways following peripheral nerve regeneration in fetal monkeys. Nature 381:69-71.

Fox K (2009) Experience-dependent plasticity mechanisms for neural rehabilitation in somatosensory cortex. Philos Trans R Soc Lond B Biol Sci 364:369-381.

Friedman RM, Chen LM, Roe AW (2008) Responses of areas $3 b$ and 1 in anesthetized squirrel monkeys to single- and dual-site stimulation of the digits. J Neurophysiol 100:3185-3196.

Frostig RD (2006) Functional organization and plasticity in the adult rat barrel cortex: moving out-of-the-box. Curr Opin Neurobiol 16:445-450.

Frostig RD, Xiong Y, Chen-Bee CH, Kvasnák E, Stehberg J (2008) Largescale organization of rat sensorimotor cortex based on a motif of large activation spreads. J Neurosci 28:13274-13284.

Fukuda M, Rajagopalan UM, Homma R, Matsumoto M, Nishizaki M, Tanifuji M (2005) Localization of activity-dependent changes in blood volume to submillimeter-scale functional domains in cat visual cortex. Cereb Cortex 15:823-833

Fukuda M, Moon CH, Wang P, Kim SG (2006) Mapping iso-orientation columns by contrast agent-enhanced functional magnetic resonance imaging: reproducibility, specificity, and evaluation by optical imaging of intrinsic signal. J Neurosci 26:11821-11832.

Gallyas F (1979) Silver staining of myelin by means of physical development. Neurol Res 1:203-209.

Garraghty PE, LaChica EA, Kaas JH (1991) Injury-induced reorganization of somatosensory cortex is accompanied by reductions in GABA staining. Somatosens Mot Res 8:347-354.

Garraghty PE, Arnold LL, Wellman CL, Mowery TM (2006) Receptor auto- 
radiographic correlates of deafferentation-induced reorganization in adult primate somatosensory cortex. J Comp Neurol 497:636-645.

Gilman S, Denny-Brown D (1966) Disorders of movement and behaviour following dorsal column lesions. Brain 89:397-418.

Graziano A, Jones EG (2009) Early withdrawal of axons from higher centers in response to peripheral somatosensory denervation. J Neurosci 29:3738-3748.

Grinvald A, Lieke E, Frostig RD, Gilbert CD, Wiesel TN (1986) Functional architecture of cortex revealed by optical imaging of intrinsic signals. Nature 324:361-364.

Harel N (2012) Ultra high resolution fMRI at ultra-high field. Neuroimage 62:1024-1028.

Harel N, Uğurbil K, Uludağ K, Yacoub E (2006) Frontiers of brain mapping using MRI. J Magn Reson Imaging 23:945-957.

Hill DL, Hawkes DJ, Crossman JE, Gleeson MJ, Cox TC, Bracey EE, Strong AJ, Graves P (1991) Registration of MR and CT images for skull base surgery using point-like anatomical features. Br J Radiol 64:1030-1035.

Jain N, Catania KC, Kaas JH (1997) Deactivation and reactivation of somatosensory cortex after dorsal spinal cord injury. Nature 386:495-498.

Jain N, Florence SL, Kaas JH (1998) Reorganization of somatosensory cortex after nerve and spinal cord injury. News Physiol Sci 13:143-149.

Jain N, Qi HX, Kaas JH (2001) Long-term chronic multichannel recordings from sensorimotor cortex and thalamus of primates. Prog Brain Res 130:64-72.

Jain N, Qi HX, Collins CE, Kaas JH (2008) Large-scale reorganization in the somatosensory cortex and thalamus after sensory loss in macaque monkeys. J Neurosci 28:11042-11060.

Jones EG (2000) Cortical and subcortical contributions to activitydependent plasticity in primate somatosensory cortex. Annu Rev Neurosci 23:1-37.

Kaas JH (2000) The reorganization of somatosensory and motor cortex after peripheral nerve or spinal cord injury in primates. Prog Brain Res 128:173-179.

Kaas JH, Qi HX, Burish MJ, Gharbawie OA, Onifer SM, Massey JM (2008) Cortical and subcortical plasticity in the brains of humans, primates, and rats after damage to sensory afferents in the dorsal columns of the spinal cord. Exp Neurol 209:407-416.

Karl A, Birbaumer N, Lutzenberger W, Cohen LG, Flor H (2001) Reorganization of motor and somatosensory cortex in upper extremity amputees with phantom limb pain. J Neurosci 21:3609-3618.

Lecoeur J, Wang F, Chen LM, Li R, Avison MJ, Dawant BM (2011) Automated longitudinal registration of high resolution structural MRI brain sub-volumes in non-human primates. J Neurosci Methods 202:99-108.

Leonard CM, Glendinning DS, Wilfong T, Cooper BY, Vierck CJ Jr (1992) Alterations of natural hand movements after interruption of fasciculus cuneatus in the macaque. Somatosens Mot Res 9:75-89.

Logothetis NK, Guggenberger H, Peled S, Pauls J (1999) Functional imaging of the monkey brain. Nat Neurosci 2:555-562.

Matthews PM, Honey GD, Bullmore ET (2006) Applications of fMRI in translational medicine and clinical practice. Nat Rev Neurosci 7:732-744.

Mowery TM, Garraghty PE (2009) Nerve-injury induced changes to GluR1 and GluR2/3 sub-unit expression in area 3b of adult squirrel monkeys: developmental recapitulation? Front Syst Neurosci 3:1.

Muellbacher W, Hallett M (2006) Reprogramming surviving motor cortex after stroke. New York: Oxford UP.

Mühlnickel W, Elbert T, Taub E, Flor H (1998) Reorganization of auditory cortex in tinnitus. Proc Natl Acad Sci U S A 95:10340-10343.

Padberg J, Recanzone G, Engle J, Cooke D, Goldring A, Krubitzer L (2010) Lesions in posterior parietal area 5 in monkeys result in rapid behavioral and cortical plasticity. J Neurosci 30:12918-12935.

Qi HX, Chen LM, Kaas JH (2011) Reorganization of somatosensory cortical areas $3 \mathrm{~b}$ and 1 after unilateral section of dorsal columns of the spinal cord in squirrel monkeys. J Neurosci 31:13662-13675.

Reed JL, Qi HX, Kaas JH (2011) Spatiotemporal properties of neuron response suppression in owl monkey primary somatosensory cortex when stimuli are presented to both hands. J Neurosci 31:3589-3601.

Zhang N, Wang F, Turner GH, Gore JC, Avison MJ, Chen LM (2010) Intraand inter-subject variability of high field fMRI digit maps in somatosensory area 3b of new world monkeys. Neuroscience 165:252-264. 